MATHEMATICS OF COMPUTATION

Volume 70, Number 234, Pages 555-577

S 0025-5718(00)01256-4

Article electronically published on March 24, 2000

\title{
CONVERGENCE OF RELAXATION SCHEMES TO THE EQUATIONS OF ELASTODYNAMICS
}

\author{
LAURENT GOSSE AND ATHANASIOS E. TZAVARAS
}

\begin{abstract}
We study the effect of approximation matrices to semi-discrete relaxation schemes for the equations of one-dimensional elastodynamics. We consider a semi-discrete relaxation scheme and establish convergence using the $L^{p}$ theory of compensated compactness. Then we study the convergence of an associated relaxation-diffusion system, inspired by the scheme. Numerical comparisons of fully-discrete schemes are carried out.
\end{abstract}

\section{INTRODUCTION}

Relaxation approximations of hyperbolic conservation laws appear in diverse models in continuum mechanics and kinetic theory of gases, and serve as a groundstage for the design of numerical schemes for hyperbolic systems of conservation laws (see [9, 4, 5, 23, 2, for a range of perspectives and [16] for the related subject of kinetic schemes). The convergence properties of relaxation systems and associated relaxation schemes for scalar conservation laws are presently well understood (e.g., 4, 15, 1, 21, 10,11]). By contrast, when the zero-relaxation limit is a system of conservation laws, the dissipative effect of relaxation is subtle to capture and convergence results were only recently established [22, 23, 17, 12].

The issue of dependence on the approximation matrix, familiar from the theory of viscosity approximations, has a counterpart in the theory of relaxation approximations and their associated relaxation schemes. As a test case to investigate this issue, we consider the system of one-dimensional elastodynamics,

$$
\begin{aligned}
u_{t}-v_{x} & =0, \\
v_{t}-g(u)_{x} & =0,
\end{aligned}
$$

where $u$ stands for the (shear) strain and $v$ for the velocity in the direction of the motion.

We compare two relaxation systems, both having as zero relaxation limit $\varepsilon \rightarrow 0$ the equations (1). The first system reads:

$$
\begin{aligned}
u_{t}-v_{x} & =0 \\
v_{t}-\sigma_{x} & =0 \\
(\sigma-E u)_{t} & =-\frac{1}{\varepsilon}(\sigma-g(u)) .
\end{aligned}
$$

Received by the editor March 23, 1999.

2000 Mathematics Subject Classification. Primary 35L65, 65M12.

Key words and phrases. Relaxation schemes, compensated compactness.

This joint work was partially supported by TMR project HCL \#ERBFMRXCT960033. The second author acknowledges support of the National Science Foundation and the Office for Naval Research. 
It is a model in viscoelasticity [7] 23], and it may be put into the equivalent form

$$
\begin{aligned}
u_{t}-v_{x} & =0, \\
v_{t}-g(u)_{x} & =\varepsilon\left(E v_{x x}-v_{t t}\right),
\end{aligned}
$$

of an approximation of (11) by one wave equation. The second system is of the type proposed by Jin and Xin in [9]:

$$
\begin{aligned}
u_{t}-p_{x} & =0 \\
v_{t}-r_{x} & =0 \\
p_{t}-\kappa u_{x} & =-\frac{1}{\varepsilon}(p-v), \\
r_{t}-\lambda v_{x} & =-\frac{1}{\varepsilon}(r-g(u)),
\end{aligned}
$$

and it may be written in the equivalent form

$$
\begin{aligned}
u_{t}-v_{x} & =\varepsilon\left(\kappa u_{x x}-u_{t t}\right), \\
v_{t}-g(u)_{x} & =\varepsilon\left(\lambda v_{x x}-v_{t t}\right)
\end{aligned}
$$

of an approximation of (1) by two wave equations.

For stress-strain functions $g$ satisfying $g^{\prime}>0$ the system (1) is strictly hyperbolic with characteristic speeds $\lambda_{1,2}(u)= \pm\left(g^{\prime}(u)\right)^{1 / 2}$. The zero-relaxation limit from (2) to $(\mathbb{1})$ is performed in [23] under the hypotheses $g(0)=0$,

$$
0<\gamma \leq g^{\prime}(u) \leq \Gamma, \quad u \in \mathbb{R}
$$

for some positive constants $\gamma, \Gamma$,

$$
\begin{gathered}
\left(u-u_{0}\right) g^{\prime \prime}(u) \neq 0 \quad \text { for } u \neq u_{0}, \text { and } \\
g^{\prime \prime}, g^{\prime \prime \prime} \in L^{2} \cap L^{\infty}(\mathbb{R}) .
\end{gathered}
$$

and for the parameter $E$ selected so that $E>\Gamma$. Note that (2) is a model in viscoelasticity and that the subcharacteristic condition $g^{\prime}<E$ guarantees that the model is consistent with the second law of thermodynamics 77,23 . The zerorelaxation limit from (4) to (1) is established in 17], under the following hypotheses: $g$ is stricly increasing, $g$ convex for $u>0$ and concave for $u<0$ and $\kappa=\lambda$ sufficiently large.

The objective of this article is to study the effects of different relaxation approximations on the associated numerical schemes. Each of the systems (2) and (4) can be put into diagonal form and suggests, by upwinding, very natural relaxing and relaxed schemes. For instance, upon introducing the Riemann invariants, (2) is put into the diagonal form

$$
\begin{aligned}
\partial_{t}\left(\frac{\sigma}{2 E}+\frac{v}{2 \sqrt{E}}\right)-\sqrt{E} \partial_{x}\left(\frac{\sigma}{2 E}+\frac{v}{2 \sqrt{E}}\right) & =-\frac{1}{\varepsilon 2 E}(\sigma-g(u)), \\
\partial_{t}\left(\frac{\sigma}{2 E}-\frac{v}{2 \sqrt{E}}\right)+\sqrt{E} \partial_{x}\left(\frac{\sigma}{2 E}-\frac{v}{2 \sqrt{E}}\right) & =-\frac{1}{\varepsilon 2 E}(\sigma-g(u)), \\
\partial_{t}\left(u-\frac{\sigma}{E}\right) & =-\frac{1}{\varepsilon E}(g(u)-\sigma) .
\end{aligned}
$$

Performing upwinding in (8) yields a semi-discrete scheme, for the values of $(u, v, \sigma)$ on the lattice $\mathbb{Z}$,

$$
t \mapsto\left(u_{j}(t), v_{j}(t), \sigma_{j}(t)\right)_{j \in \mathbb{Z}},
$$

that reads

$$
\begin{aligned}
\left(u_{j}\right)_{t}-\frac{1}{2 h}\left(v_{j+1}-v_{j-1}\right) & =\frac{1}{2 h \sqrt{E}}\left(\sigma_{j+1}-2 \sigma_{j}+\sigma_{j-1}\right) \\
\left(v_{j}\right)_{t}-\frac{1}{2 h}\left(\sigma_{j+1}-\sigma_{j-1}\right) & =\frac{\sqrt{E}}{2 h}\left(v_{j+1}-2 v_{j}+v_{j-1}\right) \\
\left(\sigma_{j}-E u_{j}\right)_{t} & =-\frac{1}{\varepsilon}\left(\sigma_{j}-g\left(u_{j}\right)\right)
\end{aligned}
$$


In a similar fashion, (4) is diagonalizable and has corresponding upwinding relaxed and relaxing schemes (see Jin and Xin [9]).

In Section 3, we establish convergence of the semi-discrete scheme (9) to the elasticity system, under the hypotheses ([6), (7) and $E>\Gamma$, in the parameter range $h \rightarrow 0, \varepsilon \rightarrow 0$ with $\varepsilon=O(h)$. The restriction $\varepsilon=O(h)$ does not appear in convergence results of semi-discrete schemes to scalar conservation laws [1], nor in a recent convergence result for fully disrete schemes patterned after the relaxation system (4) (see [12]). This restriction reflects the nature of the relaxation approximation, as is explained in this article.

Note that (9) is a formal first order discretization of (2). Experience with discretizations of hyperbolic systems would suggest that the behaviour of (9) is characterized by the associated relaxation-diffusion system

$$
\begin{aligned}
u_{t}-v_{x} & =h \frac{1}{2 \sqrt{E}} \sigma_{x x}, \\
v_{t}-\sigma_{x} & =h \frac{\sqrt{E}}{2} v_{x x}, \\
(\sigma-E u)_{t} & =-\frac{1}{\varepsilon}(\sigma-g(u)) .
\end{aligned}
$$

In principle, the presence of diffusion should reinforce any stabilizing effect of relaxation and provide a more stable response. Indeed, this is the case for a relaxationdiffusion system that looks exactly like (10) with the notable exception that the term $\sigma_{x x}$ is replaced by $u_{x x}$ (see Lu and Klingenberg [13]). However, for the system (10) this is not the case; the slight difference in terms makes a large difference in the analysis, which is based on using the stabilizing effect of relaxation to compensate for the missing control of incomplete diffusion matrix. We establish convergence of (10) to (1) as $\varepsilon \rightarrow 0, h \rightarrow 0$ in the parameter range $h=o(\varepsilon)$. Somewhat surprisingly, this is the opposite range from the convergence range of the relaxing semi-discrete scheme. We discuss this discrepancy at the end of Section 4.

In order to investigate whether the parameter range $\varepsilon=O(h)$ is a real restriction for convergence (of the semi-discrete scheme) or a deficiency of our analysis, we performed extensive numerical runs, comparing fully discrete schemes based on (4) with fully discrete schemes based on (2). It turns out that both discretizations are very stable in the parameter region $\varepsilon=O(h)$, but the one based on (2) develops oscillations as we move to the boundary of the parameter region (see Section 5). On the other hand, in the region that both are stable, the upwind discretization of (21) is far less diffusive than the upwind discretization of (4).

The main ingredients for both convergence results are the theory of compensated compactness (Tartar 20], Murat [14]), the $L^{p}$ theory for the reduction of generalized Young measures for the equations of elastodynamics (DiPerna [6], Shearer [19], Serre-Shearer [18]), and a priori estimates - valid under the hypothesis $0<g^{\prime}<E-$ measuring the dissipative strength of the semi-discrete relaxation scheme (9) and of the relaxation-diffusion system (10). The a priori estimates are quite different in spirit for the two cases. In the former case, diffusive effects of the semi-discrete scheme dominate the relaxation effects (using the fact that for a scheme there can be no oscillations below the scale of the grid), while, in the latter case, relaxation dominates diffusion - in the range $h=o(\varepsilon)$.

To illustrate the analytical aspects, we start in Section 2 by proving convergence for a variant of (4) (see (14) in Section 2) to the equations of elasticity in the limit 
$\varepsilon \rightarrow 0$. This result indicates the robustness of the method, relative to different approximation matrices. In all cases some version of the subcharacteristic condition must be satisfied to ensure stability and convergence.

Let us mention a different perspective that links (9) to discrete kinetic schemes (see Aregba-Driollet and Natalini [2]). When (8) is expressed in terms of the Riemann invariants

$$
f_{1}=\frac{\sigma}{2 E}+\frac{v}{2 \sqrt{E}}, \quad f_{2}=\frac{\sigma}{2 E}-\frac{v}{2 \sqrt{E}}, \quad f_{3}=u-\frac{\sigma}{E},
$$

it leads to a system for the evolution of $\left(f_{1}, f_{2}, f_{3}\right)$

$$
\begin{aligned}
\partial_{t} f_{1}-\sqrt{E} \partial_{x} f_{1} & =-\frac{1}{\varepsilon}\left(f_{1}-\mathcal{M}_{1}(u, v)\right), \\
\partial_{t} f_{2}+\sqrt{E} \partial_{x} f_{2} & =-\frac{1}{\varepsilon}\left(f_{2}-\mathcal{M}_{2}(u, v)\right), \\
\partial_{t} f_{3} & =-\frac{1}{\varepsilon}\left(f_{3}-\mathcal{M}_{3}(u, v)\right),
\end{aligned}
$$

where

$$
\mathcal{M}_{1}=\frac{g(u)}{2 E}+\frac{v}{2 \sqrt{E}}, \quad \mathcal{M}_{2}=\frac{g(u)}{2 E}-\frac{v}{2 \sqrt{E}}, \quad \mathcal{M}_{3}=u-\frac{g(u)}{E} .
$$

Then (12) may be interpreted as a discrete BGK-approximation; $u, v$ and $\sigma$ are recovered as moments

$$
u=f_{1}+f_{2}+f_{3}, \quad v=f_{1} \sqrt{E}-f_{2} \sqrt{E}, \quad \sigma=f_{1} E+f_{2} E ;
$$

and the Maxwellian functions $\mathcal{M}_{1}, \mathcal{M}_{2}, \mathcal{M}_{3}$ satisfy

$$
\begin{aligned}
u & =\mathcal{M}_{1}(u, v)+\mathcal{M}_{2}(u, v)+\mathcal{M}_{3}(u, v), \\
v & =\mathcal{M}_{1}(u, v) \sqrt{E}-\mathcal{M}_{2}(u, v) \sqrt{E} \\
g(u) & =\mathcal{M}_{1}(u, v) E+\mathcal{M}_{2}(u, v) E
\end{aligned}
$$

Therefore, (2) describes the evolution of moments for the discrete kinetic model (12), and the upwinding scheme studied here is the natural kinetic scheme for (12).

\section{Relaxation approximations}

In this section, we discuss the convergence of the relaxation system

$$
\partial_{t}\left(\begin{array}{l}
u \\
v
\end{array}\right)-\partial_{x}\left(\begin{array}{c}
v \\
g(u)
\end{array}\right)=\varepsilon A \partial_{x x}\left(\begin{array}{l}
u \\
v
\end{array}\right)-\varepsilon \partial_{t t}\left(\begin{array}{l}
u \\
v
\end{array}\right)
$$

to the equations of elasticity as $\varepsilon \rightarrow 0$. Note that (14) is an approximation of the type proposed by Jin and Xin in [9] and (4) corresponds to the special case that $A$ is diagonal. We refer to Serre 17] for a convergence result for (4) under a different methodology.

First, we state a general estimate from [23. Consider a system of $N$ conservation laws

$$
U_{t}+F(U)_{x}=0
$$

that is equipped with a strictly convex entropy $H(U)$, and let $Q(U)$ be the corresponding flux. Recall that entropy-entropy flux pairs $H-Q$ are generated by solving the system of linear hyperbolic equations

$$
Q^{\prime}=H^{\prime} \cdot F^{\prime},
$$

where $H^{\prime}=\nabla H$ stands for the gradient of $H$ and $H^{\prime \prime}$ for its Hessian matrix. Consider the relaxation approximation of (15)

$$
U_{t}+F(U)_{x}=\varepsilon\left(A U_{x x}-U_{t t}\right),
$$


where $A$ is a positive definite and symmetric matrix. We have

Proposition 1. Assume that (15) is equipped with a strictly convex entropy $H(U)$ satisfying, for some $\alpha>0$ and $\mu>0$,

$$
H^{\prime \prime}(U)-\alpha I \geq \mu I,
$$

and suppose that the positive-definite symmetric matrix A satisfies, for some $\nu>0$,

$$
\frac{1}{2}\left[\left(H^{\prime \prime}(U) A\right)^{T}+H^{\prime \prime}(U) A\right]-\alpha F^{\prime}(U)^{T} F^{\prime}(U) \geq \nu I .
$$

Then smooth solutions $U$ of (16), that decay fast at infinity, satisfy the dissipation estimate

$$
\partial_{t} e+\partial_{x} Q(U)+\varepsilon \mu\left|U_{t}\right|^{2}+\varepsilon \nu\left|U_{x}\right|^{2} \leq \partial_{x}\left(\varepsilon H^{\prime} \cdot A U_{x}+2 \varepsilon^{2} \alpha U_{t} \cdot A U_{x}\right),
$$

where

$$
\begin{aligned}
e=H & \left(U+\varepsilon U_{t}\right)+\varepsilon^{2} U_{t} \cdot\left[\frac{1}{2} \alpha I-\int_{0}^{1} \int_{0}^{s} H^{\prime \prime}\left(U+\varepsilon \tau U_{t}\right) d \tau d s\right] U_{t} \\
& +\frac{1}{2} \varepsilon^{2} \alpha\left|U_{t}\right|^{2}+\varepsilon^{2} \alpha U_{x} \cdot A U_{x} .
\end{aligned}
$$

The system of elasticity is equipped with the strictly convex entropy (the mechanical energy)

$$
\begin{aligned}
& H(u, v)=\frac{1}{2} v^{2}+G(u), \quad \text { where } \quad G(u)=\int_{0}^{u} g(\xi) d \xi, \\
& Q(u, v)=-v g(u) .
\end{aligned}
$$

Under hypothesis (6) we may select $\alpha<\min \{\gamma, 1\}$. Then (17) is fulfilled for $0<\mu<\min \{\gamma-\alpha, 1-\alpha\}$ and

$$
\frac{1}{2}\left(v^{2}+\gamma u^{2}\right) \leq \frac{1}{2} v^{2}+G(u) \leq \frac{1}{2}\left(v^{2}+\Gamma u^{2}\right) .
$$

Now $\alpha$ is fixed and (18) becomes

$$
\frac{1}{2}\left(A\left(\begin{array}{ll}
g^{\prime} & 0 \\
0 & 1
\end{array}\right)+\left(\begin{array}{ll}
g^{\prime} & 0 \\
0 & 1
\end{array}\right) A\right)-\alpha\left(\begin{array}{ll}
\left(g^{\prime}\right)^{2} & 0 \\
0 & 1
\end{array}\right) \geq \nu I .
$$

If (6) holds, then (21) can always be fulfilled by selecting $A$. Proposition 1 yields for solutions $(u, v)$ of (14)

$$
\mathcal{E}(t)+\varepsilon \int_{0}^{t} \int_{\mathbb{R}} u_{t}^{2}+v_{t}^{2}+u_{x}^{2}+v_{x}^{2} d x d \tau \leq O(1) \mathcal{E}(0),
$$

where $O(1)$ stands for a constant independent of $\varepsilon$, and

$$
\mathcal{E}(t):=\int_{\mathbb{R}} u^{2}+v^{2}+\varepsilon^{2}\left(u_{t}^{2}+v_{t}^{2}+u_{x}^{2}+v_{x}^{2}\right) d x .
$$

These estimates suffice to apply the $L^{p}$ theory of compensated compactness and deduce:

Theorem 1. Let $g \in C^{3}$ satisfy (6) and (7). Let $\left(u^{\varepsilon}, v^{\varepsilon}, \sigma^{\varepsilon}\right)$ be a family of smooth solutions of (14) on $\mathbb{R} \times[0, \infty)$ emanating from smooth initial data subject to the 
$\varepsilon$-independent bounds

$$
\begin{aligned}
\mathcal{E}(0):= & \int_{\mathbb{R}} u_{0}^{\varepsilon^{2}}+v_{0}^{\varepsilon^{2}} d x \\
& +\varepsilon^{2} \int_{\mathbb{R}} u_{t}^{\varepsilon}(x, 0)^{2}+v_{t}^{\varepsilon}(x, 0)^{2}+u_{0 x}^{\varepsilon}{ }^{2}+v_{0 x}^{\varepsilon}{ }^{2} d x \leq O(1),
\end{aligned}
$$

and let $A$ be a symmetric, positive-definite matrix subject to (21). Then, along a subsequence if necessary,

$$
u^{\varepsilon} \rightarrow u, \quad v^{\varepsilon} \rightarrow v, \quad \text { a.e. }(x, t) \text { and in } L_{l o c}^{p}(\mathbb{R} \times(0, T)), \text { for } p<2,
$$

and $(u, v)$ is a weak solution of (11).

Proof. Let $\left(u^{\varepsilon}, v^{\varepsilon}\right)$ be a family of solutions to (14). The proof uses the theory of compensated compactness 20]. Typically, in such proofs, the goal is to control the dissipation measure and to show

$$
\partial_{t} \eta\left(u^{\varepsilon}, v^{\varepsilon}\right)+\partial_{x} q\left(u^{\varepsilon}, v^{\varepsilon}\right) \text { lies in a compact of } H_{l o c}^{-1}
$$

for a class of entropy-entropy flux pairs $\eta-q$ for the equations of elasticity. In the presence of uniform $L^{\infty}$-bounds, the theorem of DiPerna [6] guarantees compactness of approximate solutions and implies that, along a subsequence, $u^{\varepsilon} \rightarrow u$ and $v^{\varepsilon} \rightarrow v$ a.e. $(x, t)$.

In the present case $L^{\infty}$-estimates are only available in the special case that $A$ is a multiple of the identity matrix (see [17]) and, in view of (22), the natural stability framework is in the energy norm. Convergence of viscosity approximations to the equations of elastodynamics in the energy framework is carried out in Shearer [19] (for the genuine-nonlinear case) and Serre-Shearer [18 (for loss of genuinenonlinearity at one point). In [19] two classes of entropies, with growth controlled by the wave-speeds at infinity, are constructed ([19] Lemma 2) for which Tartar's commutation relation is justified (Lemma 3 ) and are used to show that the support of the (generalized) Young measure is a point mass (Lemma 7 and Theorem 1(iii)). When $\sigma(u)$ has one inflection point, the reduction of the Young measure is performed in [18] Lemma 3 and Section 5.

To ensure the dissipation estimate, we are operating under (6). It then it suffices to establish (23) for entropy pairs $\eta-q$ satisfying

$$
\eta, q, \eta_{u}, \eta_{v}, \eta_{u u}, \eta_{u v}, \eta_{v v} \in L^{\infty}\left(\mathbb{R}^{2}\right) .
$$

This class of entropy pairs contains (under the auspices of (6) the test-pairs that are used in 19, 18 in order to prove the reduction of the generalized Young measure to a point mass and to show strong convergence in $L_{l o c}^{p}$ for $p<2$. Hypothesis (7) reflects the assumptions needed in those works.

To complete the proof, we prove (23) for entropy entropy-flux pair $\eta-q$ satisfying (24). From (14) we have

$$
\begin{aligned}
\partial_{t} \eta\left(u^{\varepsilon}, v^{\varepsilon}\right)+\partial_{x} q\left(u^{\varepsilon}, v^{\varepsilon}\right)= & \varepsilon \partial_{x}\left(\eta^{\prime} \cdot A\left(\begin{array}{c}
u_{x} \\
v_{x}
\end{array}\right)\right)-\varepsilon \partial_{t}\left(\eta^{\prime} \cdot\left(\begin{array}{c}
u_{t} \\
v_{t}
\end{array}\right)\right) \\
& \quad-\varepsilon\left(u_{x} v_{x}\right) \cdot \eta^{\prime \prime} A\left(\begin{array}{c}
u_{x} \\
v_{x}
\end{array}\right)+\varepsilon\left(u_{t} v_{t}\right) \cdot \eta^{\prime \prime}\left(\begin{array}{c}
u_{t} \\
v_{t}
\end{array}\right) \\
= & : I_{1}+I_{2}+I_{3}+I_{4} .
\end{aligned}
$$


From (22) and (24), the terms $I_{1}, I_{2}$ lie in a compact of $H^{-1}$, the terms $I_{3}, I_{4}$ are bounded in $L^{1}$, and the sum $\sum I_{i}$ lies in a bounded set of $W^{-1, \infty}$. Murat's lemma 14] implies that (23) holds, and this concludes the proof.

\section{Convergence of UPWIND SEMi-Discrete SCHEMES}

3.1. Derivation of a numerical scheme. In this paragraph, we construct stable numerical approximations for solutions of system (2) by a natural upwinding treatment of the linear convective part. We place the hypotheses $g(0)=0$ and (6) on the function $g$, which ensure strict hyperbolicity for (11). We introduce a regular grid on the real axis with a uniform space-step denoted by $h>0$. It is expedient to formulate the problem in terms of the Riemann invariants

$$
v \pm \frac{\sigma}{\sqrt{E}} \text { and } \sigma-E u
$$

Then (2) is put into diagonal form:

$$
\begin{aligned}
\left(v+\frac{\sigma}{\sqrt{E}}\right)_{t}-\sqrt{E}\left(v+\frac{\sigma}{\sqrt{E}}\right)_{x} & =-\frac{1}{\sqrt{E} \varepsilon}(\sigma-g(u)), \\
\left(v-\frac{\sigma}{\sqrt{E}}\right)_{t}+\sqrt{E}\left(v-\frac{\sigma}{\sqrt{E}}\right)_{x} & =\frac{1}{\sqrt{E} \varepsilon}(\sigma-g(u)), \\
(\sigma-E u)_{t} & =-\frac{1}{\varepsilon}(\sigma-g(u)) .
\end{aligned}
$$

We derive a numerical approximation by means of the following semi-discrete algorithm, which consists in an upwind treatment of the characteristic variables (whose propagation speeds are fixed):

$$
\begin{aligned}
\left(v_{j}+\frac{\sigma_{j}}{\sqrt{E}}\right)_{t}-\frac{\sqrt{E}}{h}\left[\left(v_{j+1}+\frac{\sigma_{j+1}}{\sqrt{E}}\right)-\left(v_{j}+\frac{\sigma_{j}}{\sqrt{E}}\right)\right] & =-\frac{1}{\sqrt{E} \varepsilon}\left(\sigma_{j}-g\left(u_{j}\right)\right), \\
\left(v_{j}-\frac{\sigma_{j}}{\sqrt{E}}\right)_{t}+\frac{\sqrt{E}}{h}\left[\left(v_{j}-\frac{\sigma_{j}}{\sqrt{E}}\right)-\left(v_{j-1}-\frac{\sigma_{j-1}}{\sqrt{E}}\right)\right] & =\frac{1}{\sqrt{E} \varepsilon}\left(\sigma_{j}-g\left(u_{j}\right)\right), \\
\left(\sigma_{j}-E u_{j}\right)_{t} & =-\frac{1}{\varepsilon}\left(\sigma_{j}-g\left(u_{j}\right)\right) .
\end{aligned}
$$

Let $t \mapsto\left(u_{j}(t), v_{j}(t), \sigma_{j}(t)\right)_{j \in \mathbb{Z}}$ denote the solution of the preceding differential system on the lattice $\mathbb{Z}$ for $t>0$. The subscript $j$ means that the quantity is an approximation of the average of the exact solution of (2) in the interval centered at $x_{j}=j h$ of length $h$. The system may be rewritten as

$$
\left\{\begin{array}{l}
\left(\frac{\sigma_{j}}{\sqrt{E}}\right)_{t}-\frac{\sqrt{E}}{2 h}\left(v_{j+1}-v_{j-1}\right)-\frac{1}{2 h}\left(\sigma_{j+1}-2 \sigma_{j}+\sigma_{j-1}\right)=\frac{-\left(\sigma_{j}-g\left(u_{j}\right)\right)}{\sqrt{E} \varepsilon}, \\
\left(v_{j}\right)_{t}-\frac{1}{2 h}\left(\sigma_{j+1}-\sigma_{j-1}\right)-\frac{\sqrt{E}}{2 h}\left(v_{j+1}-2 v_{j}+v_{j-1}\right)=0, \\
\left(\sigma_{j}-E u_{j}\right)_{t}=-\frac{1}{\varepsilon}\left(\sigma_{j}-g\left(u_{j}\right)\right) .
\end{array}\right.
$$

It is convenient to replace the first equation by

$$
\left(u_{j}\right)_{t}-\frac{1}{2 h}\left(v_{j+1}-v_{j-1}\right)-\frac{1}{2 h \sqrt{E}}\left(\sigma_{j+1}-2 \sigma_{j}+\sigma_{j-1}\right)=0 .
$$

The classical theory of differential equations in Banach spaces implies that, for fixed $h, \varepsilon>0$ and for initial data $\left(v_{j}(0), u_{j}(0), \sigma_{j}(0)\right)$ in $\ell^{\infty}(\mathbb{Z})$, there exists a unique solution of (25),

$$
\left(u_{j}, v_{j}, \sigma_{j}\right) \in C^{1}\left([0,+\infty), \ell^{\infty}(\mathbb{Z})\right)
$$


This is a consequence of the Cauchy-Lipschitz theorem (cf. [3] p. 104), since each equation in (25) depends on a finite number of values.

We define for each $h>0, \varepsilon>0$ approximations of (2) denoted by

$$
\left(u^{h, \varepsilon}, v^{h, \varepsilon}, \sigma^{h, \varepsilon}\right) \in C^{1}\left([0,+\infty), L^{\infty}(\mathbb{R})\right)
$$

such that

$$
\begin{gathered}
\left(u^{h, \varepsilon}, v^{h, \varepsilon}, \sigma^{h, \varepsilon}\right)(x, t):=\left(u_{j}(t), v_{j}(t), \sigma_{j}(t)\right) \\
\text { for } x \in K_{j}=\left[\left(j-\frac{1}{2}\right) h,\left(j+\frac{1}{2}\right) h\right), t \in \mathbb{R}^{+} .
\end{gathered}
$$

The goal is to prove compactness of the sequence of approximations as $\varepsilon$ and $h$ are sent to zero with $\varepsilon=O(h)$.

3.2. Energy and relaxation estimates. In this paragraph, we work with the upwind discretization (25). Following the ideas previously introduced for the continuous problem [23], we first prove

Lemma 1. Let $\left(u_{0}^{h, \varepsilon}, v_{0}^{h, \varepsilon}, \sigma_{0}^{h, \varepsilon}\right)$ be uniformly bounded in $L^{2}(\mathbb{R})$. If $0<g^{\prime}<E$, then we have the following bounds for all $T>0$ :

- $\left\|u^{h, \varepsilon}(., T)\right\|_{L^{2}(\mathbb{R})}+\left\|v^{h, \varepsilon}(., T)\right\|_{L^{2}(\mathbb{R})}+\left\|\sigma^{h, \varepsilon}(., T)\right\|_{L^{2}(\mathbb{R})} \leq O(1)$,

- $\left\|\tau_{h}\left(v^{h, \varepsilon}\right)-v^{h, \varepsilon}\right\|_{L^{2}([0, T] \times \mathbb{R})}^{2}+\left\|\tau_{h}\left(\sigma^{h, \varepsilon}\right)-\sigma^{h, \varepsilon}\right\|_{L^{2}([0, T] \times \mathbb{R})}^{2} \leq O(h)$,

- $\left\|\sigma^{h, \varepsilon}-g\left(u^{h, \varepsilon}\right)\right\|_{L^{2}([0, T] \times \mathbb{R})}^{2} \leq O(\varepsilon)$,

where $\tau_{h}$ stands for the $h$-translation operator

$$
\tau_{h}\left(v^{h, \varepsilon}\right)(., t)=v^{h, \varepsilon}(.+h, t) .
$$

Proof. The viscoelastic model (2) is endowed with a free energy function

$$
\psi(u, \sigma)=\frac{\sigma^{2}}{2 E}+\phi(\sigma-E u), \quad \text { where } \phi^{\prime}(\alpha)=-\frac{\alpha}{E}-h^{-1}(\alpha),
$$

and $h^{-1}$ is the inverse function of $h(u)=g(u)-E u$. For $0<g^{\prime}<E$, the function $\phi$ is well defined and has the properties

$$
\begin{gathered}
0<\frac{\gamma}{E(E-\gamma)} \leq \phi^{\prime \prime}(\alpha)=\frac{g^{\prime}(u)}{E\left(E-g^{\prime}(u)\right)} \leq \frac{\Gamma}{E(E-\Gamma)}, \\
\left(u-h^{-1}(\alpha)\right)(\alpha-h(u)) \geq \frac{1}{E}(\alpha-h(u))^{2} .
\end{gathered}
$$

Multiplying the three ordinary differential equations of the scheme (25) by $\frac{\sigma_{j}}{\sqrt{E}}$, $v_{j}, \phi^{\prime}\left(\sigma_{j}-E u_{j}\right)$, respectively, and adding yields

$$
\begin{aligned}
\frac{d}{d t}( & \left.\frac{\left(v_{j}\right)^{2}}{2}+\frac{\left(\sigma_{j}\right)^{2}}{2 E}+\phi\left(\sigma_{j}-E u_{j}\right)\right) \\
& -\frac{\sigma_{j}}{2 h}\left(v_{j+1}-v_{j-1}\right)-\frac{1}{2 h \sqrt{E}}\left[\sigma_{j}\left(\sigma_{j+1}-\sigma_{j}\right)-\sigma_{j}\left(\sigma_{j}-\sigma_{j-1}\right)\right] \\
& -\frac{v_{j}}{2 h}\left(\sigma_{j+1}-\sigma_{j-1}\right)-\frac{\sqrt{E}}{2 h}\left[v_{j}\left(v_{j+1}-v_{j}\right)-v_{j}\left(v_{j}-v_{j-1}\right)\right] \\
& +\frac{1}{\varepsilon}\left[\frac{\sigma_{j}}{E}+\phi^{\prime}\left(\sigma_{j}-E u_{j}\right)\right]\left(\sigma_{j}-g\left(u_{j}\right)\right)=0
\end{aligned}
$$


From (29),

$$
\left(\frac{\sigma_{j}}{E}+\phi^{\prime}\left(\sigma_{j}-E u_{j}\right)\right)\left(\sigma_{j}-g\left(u_{j}\right)\right) \geq \frac{1}{E}\left(\sigma_{j}-g\left(u_{j}\right)\right)^{2} .
$$

Summing over $j \in \mathbb{Z}$ and using

$$
\frac{1}{2 h} \sum_{j \in \mathbb{Z}} h\left[v_{j}\left(\sigma_{j+1}-\sigma_{j-1}\right)+\sigma_{j}\left(v_{j+1}-v_{j-1}\right)\right]=0,
$$

we obtain

$$
\begin{aligned}
\frac{d}{d t} \sum_{j \in \mathbb{Z}} h & {\left[\frac{\left(v_{j}\right)^{2}}{2}+\psi\left(u_{j}, \sigma_{j}\right)\right]+\frac{\sqrt{E}}{2 h} \sum_{j \in \mathbb{Z}} h\left(v_{j+1}-v_{j}\right)^{2} } \\
+ & \frac{1}{2 h \sqrt{E}} \sum_{j \in \mathbb{Z}} h\left(\sigma_{j+1}-\sigma_{j}\right)^{2}+\frac{1}{\varepsilon E} \sum_{j \in \mathbb{Z}} h\left(\sigma_{j}-g\left(u_{j}\right)\right)^{2} \leq 0 .
\end{aligned}
$$

As a first consequence, we have for $t>0$

$$
\sum_{j \in \mathbb{Z}} h\left[\frac{\left(v_{j}(t)\right)^{2}}{2}+\psi\left(u_{j}(t), \sigma_{j}(t)\right)\right] \leq \sum_{j \in \mathbb{Z}} h\left[\frac{\left(v_{j}(0)\right)^{2}}{2}+\psi\left(u_{j}(0), \sigma_{j}(0)\right)\right] .
$$

Since $\frac{v^{2}}{2}+\psi(u, \sigma)$ is equivalent to the $L^{2}$ norm, this inequality implies the energy estimate (28) 1 and the bounds

$$
\int_{0}^{t} \int_{\mathbb{R}}\left(\tau_{h}\left(v^{h, \varepsilon}\right)-v^{h, \varepsilon}\right)^{2}+\left(\tau_{h}\left(\sigma^{h, \varepsilon}\right)-\sigma^{h, \varepsilon}\right)^{2} d x d t \leq O(h)
$$

and

$$
\int_{0}^{t} \int_{\mathbb{R}}\left[v^{h, \varepsilon}(x, s)-g\left(u^{h, \varepsilon}\right)(x, s)\right]^{2} d x d s \leq O(\varepsilon)
$$

for all $t>0$.

Lemma 2. Under the hypotheses of Lemma 1, we have for $T>0$

$$
\left\|\tau_{h}\left(u^{h, \varepsilon}\right)-u^{h, \varepsilon}\right\|_{L^{2}([0, T] \times \mathbb{R})}^{2} \leq O(h)+O(\varepsilon) .
$$

Proof. Using the identity

$$
\tau_{h}\left(g\left(u^{h, \varepsilon}\right)\right)-g\left(u^{h, \varepsilon}\right)=\tau_{h}\left(g\left(u^{h, \varepsilon}\right)-\sigma^{h, \varepsilon}\right)+\left(\tau_{h}\left(\sigma^{h, \varepsilon}\right)-\sigma^{h, \varepsilon}\right)+\left(\sigma^{h, \varepsilon}-g\left(u^{h, \varepsilon}\right)\right)
$$

and the estimates in (28), we derive the desired result.

Remark 1. Lemma 2 shows that control of the numerical derivative for $\sigma^{h, \varepsilon}$ and the distance from equilibrium $\sigma^{h, \varepsilon}-g\left(u^{h, \varepsilon}\right)$ entails control on the numerical derivative of $g\left(u^{h, \varepsilon}\right)$. Such a result is clearly false for actual derivatives, and is due to the fact that for numerical approximations there are no oscillations below the scale of the grid. 
3.3. Entropy consistency and convergence. In order to use the $L^{2}$ theory of approximate solutions for (11) [19, 18], under the framework of ([6), we need control of the entropy dissipation for entropy pairs $\eta-q$ satisfying the growth restrictions (24) (cf. Theorem प).

Lemma 3. Under the hypotheses of Lemma 1, if $\varepsilon=O(h)$, then

$$
\eta\left(u^{h, \varepsilon}, v^{h, \varepsilon}\right)_{t}+q\left(u^{h, \varepsilon}, v^{h, \varepsilon}\right)_{x} \text { lies in a compact of } H_{\text {loc }}^{-1}\left(\mathbb{R} \times \mathbb{R}^{+}\right)
$$

for any entropy-entropy flux pair $\eta-q$ satisfying (24).

Proof. We introduce the notations

$$
\begin{aligned}
& D_{c}^{h}\left(\sigma_{j}\right)=\left(\sigma_{j+1}-\sigma_{j-1}\right) / 2 h, \\
& \Delta^{h}\left(\sigma_{j}\right)=\left(\sigma_{j+1}-2 \sigma_{j}+\sigma_{j-1}\right) / h^{2},
\end{aligned}
$$

and write the scheme in the form

$$
\begin{aligned}
\left(u_{j}\right)_{t}-D_{c}^{h}\left(v_{j}\right)-\frac{h}{2 \sqrt{E}} \Delta^{h}\left(\sigma_{j}\right) & =0, \\
\left(v_{j}\right)_{t}-D_{c}^{h}\left(g\left(u_{j}\right)\right)-\frac{h \sqrt{E}}{2} \Delta^{h}\left(v_{j}\right) & =D_{c}^{h}\left(\sigma_{j}-g\left(u_{j}\right)\right) .
\end{aligned}
$$

Let $K_{j}$ denote the cell $\left[\left(j-\frac{1}{2}\right) h,\left(j+\frac{1}{2}\right) h\right)$. A computation shows that

$$
\partial_{t} \eta\left(u^{h, \varepsilon}, v^{h, \varepsilon}\right)+\partial_{x} q\left(u^{h, \varepsilon}, v^{h, \varepsilon}\right)=A+B+C,
$$

where

$$
\begin{aligned}
A & =\partial_{x} q\left(u^{h, \varepsilon}, v^{h, \varepsilon}\right)+\sum_{j \in \mathbb{Z}}\left[\left(\eta_{u}\right)_{j} D_{c}^{h}\left(v_{j}\right)+\left(\eta_{v}\right)_{j} D_{c}^{h}\left(g\left(u_{j}\right)\right)\right] \mathbb{1}_{K_{j}}, \\
B & =\frac{1}{2 \sqrt{E}} \sum_{j \in \mathbb{Z}} h\left(\eta_{u}\right)_{j} \Delta^{h}\left(\sigma_{j}\right) \mathbb{1}_{K_{j}}+\frac{\sqrt{E}}{2} \sum_{j \in \mathbb{Z}} h\left(\eta_{v}\right)_{j} \Delta^{h}\left(v_{j}\right) \mathbb{1}_{K_{j}} \\
& =\frac{1}{2 \sqrt{E}} B_{1}+\frac{\sqrt{E}}{2} B_{2}, \\
C & =\sum_{j \in \mathbb{Z}}\left(\eta_{v}\right)_{j} D_{c}^{h}\left(\sigma_{j}-g\left(u_{j}\right)\right) \mathbb{1}_{K_{j}},
\end{aligned}
$$

and we used the notation

$$
\left(\eta_{u}\right)_{j}=\eta_{u}\left(u_{j}, v_{j}\right), \quad\left(\eta_{v}\right)_{j}=\eta_{v}\left(u_{j}, v_{j}\right) .
$$

Now $A, B, C$ are considerered as elements of $\mathcal{D}^{\prime}\left(\mathbb{R} \times \mathbb{R}^{+}\right)$and are estimated for $\varepsilon=O(h)$. For instance, for $\varphi \in \mathcal{D}(\mathbb{R} \times(0, \infty))$ a test function, we have

$$
\begin{aligned}
\left\langle B_{1}, \varphi\right\rangle= & \int_{\mathbb{R}^{+}} \sum_{j \in \mathbb{Z}} h\left(\eta_{u}\right)_{j} \Delta^{h}\left(\sigma_{j}\right) \int_{K_{j}} \varphi d x d t \\
\leq & \left\|\eta_{u}\right\|_{L^{\infty}}\left\|\tau_{h}\left(\sigma^{h, \varepsilon}\right)-\sigma^{h, \varepsilon}\right\|_{L^{2}}\left\|\varphi_{x}\right\|_{L^{2}} \\
& \quad+\frac{1}{h}\left\|\tau_{h}\left(\sigma^{h, \varepsilon}\right)-\sigma^{h, \varepsilon}\right\|_{L^{2}}\left\|\tau_{h}\left(\eta_{u}\right)-\eta_{u}\right\|_{L^{2}}\|\varphi\|_{C^{0}}
\end{aligned}
$$


and

$$
\begin{aligned}
\langle C, \varphi\rangle= & \int_{\mathbb{R}^{+}} \sum_{j \in \mathbb{Z}}\left(\eta_{v}\right)_{j} D_{c}^{h}\left(\sigma_{j}-g\left(u_{j}\right)\right) \int_{K_{j}} \varphi d x d t \\
\leq & \left\|\eta_{v}\right\|_{L^{\infty}}\left\|\sigma^{h, \varepsilon}-g\left(u^{h, \varepsilon}\right)\right\|_{L^{2}}\left\|\varphi_{x}\right\|_{L^{2}} \\
& \quad+\frac{1}{h}\left\|\sigma^{h, \varepsilon}-g\left(u^{h, \varepsilon}\right)\right\|_{L^{2}}\left\|\tau_{h}\left(\eta_{v}\right)-\eta_{v}\right\|_{L^{2}}\|\varphi\|_{C^{0}} .
\end{aligned}
$$

Therefore, $B$ and $C$ are split into two terms: one going to zero in $H^{-1}$, and one that is bounded in measures.

Since $\left(u^{h, \varepsilon}, v^{h, \varepsilon}\right)$ are piecewise constant functions, $q\left(u^{h, \varepsilon}, v^{h, \varepsilon}\right)_{x}$ is a countable sum of Dirac masses. We split the centered differences into the average of two upwind differences, $A=\frac{1}{2}\left[A^{+}+A^{-}\right]$, where the terms $A^{ \pm}$read

$$
\begin{aligned}
A^{+}=\sum_{j \in \mathbb{Z}}\left[q\left(u_{j+1}, v_{j+1}\right)-q\left(u_{j}, v_{j}\right)\right] \delta_{\left(j+\frac{1}{2}\right) h} \\
\quad+\frac{1}{h}\left[\left(\eta_{u}\right)_{j}\left(v_{j+1}-v_{j}\right)+\left(\eta_{v}\right)_{j}\left(g\left(u_{j+1}\right)-g\left(u_{j}\right)\right)\right] \mathbb{1}_{K_{j}}, \\
A^{-=} \sum_{j \in \mathbb{Z}}\left[q\left(u_{j}, v_{j}\right)-q\left(u_{j-1}, v_{j-1}\right)\right] \delta_{\left(j-\frac{1}{2}\right) h} \\
\quad+\frac{1}{h}\left[\left(\eta_{u}\right)_{j}\left(v_{j}-v_{j-1}\right)+\left(\eta_{v}\right)_{j}\left(g\left(u_{j}\right)-g\left(u_{j-1}\right)\right)\right] \mathbb{1}_{K_{j}} .
\end{aligned}
$$

By the mean-value theorem we can linearize the jumps of $q$ and $g$. For a test function $\varphi \in \mathcal{D}\left(\mathbb{R} \times \mathbb{R}^{+}\right)$, we have

$$
\begin{aligned}
&\left\langle A^{+}, \varphi\right\rangle=\int_{\mathbb{R}^{+}}\left\{\sum _ { j \in \mathbb { Z } } \left[\eta_{u}\left(u_{j}, v_{j}\right) \int_{K_{j}} \varphi(x, t) d x\right.\right. \\
&\left.\quad+q_{v}\left(\xi_{j+\frac{1}{2}}\right) \int_{K_{j}} \varphi\left(x_{j+\frac{1}{2}}, t\right) d x\right] \frac{v_{j+1}-v_{j}}{h} \\
&+\sum_{j \in \mathbb{Z}}\left[\eta_{v}\left(u_{j}, v_{j}\right) g^{\prime}\left(\zeta_{j+\frac{1}{2}}\right) \int_{K_{j}} \varphi(x, t) d x\right. \\
&\left.\left.\quad+q_{u}\left(\xi_{j+\frac{1}{2}}\right) \int_{K_{j}} \varphi\left(x_{j+\frac{1}{2}}, t\right) d x\right] \frac{u_{j+1}-u_{j}}{h}\right\} d t .
\end{aligned}
$$

Using the entropy/entropy flux compatibility gives

$$
\begin{aligned}
\left\langle A^{+}, \varphi\right\rangle=\int_{\mathbb{R}^{+}}\left\{\sum _ { j \in \mathbb { Z } } \left[\left(\eta_{u}\left(u_{j}, v_{j}\right)-\eta_{u}\left(\xi_{j+\frac{1}{2}}\right)\right) \int_{K_{j}} \varphi(x, t) d x\right.\right. & \left.+\eta_{u}\left(\xi_{j+\frac{1}{2}}\right) \int_{K_{j}} \varphi(x, t)-\varphi\left(x_{j+\frac{1}{2}}, t\right) d x\right] \frac{v_{j+1}-v_{j}}{h} \\
& +\sum_{j \in \mathbb{Z}}\left[\left(\eta_{v}\left(\zeta_{j+\frac{1}{2}}\right) g^{\prime}\left(\zeta_{j+\frac{1}{2}}\right)-\eta_{v}\left(\xi_{j+\frac{1}{2}}\right) g^{\prime}\left(\xi_{j+\frac{1}{2}}\right)\right) \int_{K_{j}} \varphi(x, t) d x\right. \\
+ & \left.\eta_{v}\left(\xi_{j+\frac{1}{2}}\right) g^{\prime}\left(\xi_{j+\frac{1}{2}}\right)\right) \int_{K_{j}} \varphi(x, t)-\varphi\left(x_{j+\frac{1}{2}}, t\right) d x \\
& \left.\left.+\left(\eta_{v}\left(u_{j}, v_{j}\right)-\eta_{v}\left(\zeta_{j+\frac{1}{2}}\right)\right) g^{\prime}\left(\zeta_{j+\frac{1}{2}}\right) \int_{K_{j}} \varphi(x, t) d x\right] \frac{u_{j+1}-u_{j}}{h}\right\} d t .
\end{aligned}
$$


This implies

$$
\begin{aligned}
\left\langle A^{+}, \varphi\right\rangle \leq \frac{\|\varphi\|_{C^{0}}}{h}\{\| & \tau_{h}\left(\eta_{u}\right)-\eta_{u}\left\|_{L^{2}}\right\| \tau_{h}\left(v^{h, \varepsilon}\right)-v^{h, \varepsilon}\left\|_{L^{2}}+\right\| \tau_{h}\left(u^{h, \varepsilon}\right)-u^{h, \varepsilon} \|_{L^{2}} \\
& \left.\times\left(\left\|\tau_{h}\left(\eta_{v} . g\right)-\eta_{v} . g\right\|_{L^{2}}+\left\|g^{\prime}\right\|_{L^{\infty}}\left\|\tau_{h}\left(\eta_{v}\right)-\eta_{v}\right\|_{L^{2}}\right)\right\} \\
+ & {\left[\left\|\eta_{u}\right\|_{L^{\infty}}\left\|\tau_{h}\left(v^{h, \varepsilon}\right)-v^{h, \varepsilon}\right\|_{L^{2}}\right.} \\
+ & \left.\left\|\eta_{v}\right\|_{L^{\infty}}\left\|g^{\prime}\right\|_{L^{\infty}}\left\|\tau_{h}\left(u^{h, \varepsilon}\right)-u^{h, \varepsilon}\right\|_{L^{2}}\right]\left\|\varphi_{x}\right\|_{L^{2}} .
\end{aligned}
$$

Then (24), in conjunction with Lemmas 1 and 2, ensures that

$$
\left\langle A^{+}, \varphi\right\rangle \leq O(1)\|\varphi\|_{C^{0}}+O(\sqrt{h})\left\|\varphi_{x}\right\|_{L^{2}} .
$$

Similar estimates hold for $A^{-}$. The statement follows from Murat's lemma [14].

We conclude as in the proof of Theorem 1:

Theorem 2. Let $\left(u_{0}^{h, \varepsilon}, v_{0}^{h, \varepsilon}, \sigma_{0}^{h, \varepsilon}\right)$ be uniformly bounded in $L^{2}(\mathbb{R})$, let $g$ satisfy (6), (77) and $E>\Gamma$. Consider the family $\left(v^{h, \varepsilon}, u^{h, \varepsilon}, \sigma^{h, \varepsilon}\right)$ of numerical approximations of (9). If $\varepsilon, h \rightarrow 0$ with $\varepsilon=O(h)$, then, along a subsequence,

$$
u^{h, \varepsilon} \rightarrow u, \quad v^{h, \varepsilon} \rightarrow v, \text { a.e. }(x, t) \text { and in } L_{l o c}^{p}(\mathbb{R} \times(0, T)) \text {, for } p<2,
$$

and $(u, v)$ is a weak solution of (11).

\section{Convergence of Relaxation-Diffusion approximations}

In this section, we study the relaxation-diffusion system

$$
\begin{array}{r}
u_{t}-v_{x}=\frac{h}{a} \sigma_{x x}, \\
v_{t}-\sigma_{x}=a h v_{x x}, \\
(\sigma-E u)_{t}=-\frac{1}{\varepsilon}(\sigma-g(u)),
\end{array}
$$

inspired by the semi-discrete numerical scheme (91). Comparing to (92), the quantity $a$ stands for $\sqrt{E}$ and $h$ stands for half of the cell width. Note that (32) approximates the equations of elasticity when $\varepsilon$ and $h$ are small:

$$
\begin{aligned}
& u_{t}-v_{x}=\frac{h}{a} \sigma_{x x}, \\
& v_{t}-g(u)_{x}=h a v_{x x}+[\sigma-g(u)]_{x},
\end{aligned}
$$

where

$$
\begin{aligned}
(\sigma-g(u))_{x} & =-\varepsilon(\sigma-E u)_{x t} \\
& =\varepsilon\left(a^{2} v_{x x}+2 h a v_{x x t}-h^{2} a^{2} v_{x x x x}-v_{t t}\right) .
\end{aligned}
$$

Hypotheses (6) and (7) are imposed on the function $g$. 
The system (32) may be written as a reaction-diffusion system

$$
\left\{\begin{array}{l}
\frac{1}{E} \sigma_{t}-v_{x}=\frac{h}{a} \sigma_{x x}-\frac{1}{\varepsilon E}(\sigma-g(u)), \\
v_{t}-\sigma_{x}=h a v_{x x} \\
(\sigma-E u)_{t}=-\frac{1}{\varepsilon}(\sigma-g(u)) .
\end{array}\right.
$$

Due to (6), the reaction term in (34) is globally Lipshitz and global existence follows from general semigroup theory (e.g., Henry [8]). If the data $v_{0}, \sigma_{0} \in H^{2}(\mathbb{R})$, $u_{0} \in H^{1}(\mathbb{R})$, then for any $T>0$ there exists a unique globally defined solution

$$
v, \sigma \in C\left([0, T], H^{2}(\mathbb{R})\right), \quad u \in C\left([0, T], H^{1}(\mathbb{R})\right) .
$$

We assume that the data satisfy the uniform bounds

$$
\begin{gathered}
\int_{\mathbb{R}}\left(u_{0}^{\varepsilon, h}\right)^{2}+\left(v_{0}^{\varepsilon, h}\right)^{2}+\left(\sigma_{0}^{\varepsilon, h}\right)^{2} d x \leq O(1), \\
\varepsilon^{2} \int_{\mathbb{R}}\left(u_{0 x}^{\varepsilon, h}\right)^{2}+\left(v_{0 x}^{\varepsilon, h}\right)^{2}+\left(\sigma_{0 x}^{\varepsilon, h}\right)^{2}+h^{2}\left(u_{0 x x}^{\varepsilon, h}\right)^{2} d x \leq O(1),
\end{gathered}
$$

and proceed to study the compactness of the family of solutions

$$
\left\{\left(u^{\varepsilon, h}, v^{\varepsilon, h}, \sigma^{\varepsilon, h}\right)\right\}_{\varepsilon, h>0}
$$

as $h$ and $\varepsilon$ are sent to zero. For notational simplicity we drop the superscripts. In the course of the proof we use estimates that require additional smoothness for their derivation. This can be removed by using standard density arguments.

4.1. Energy estimates on the relaxing approximations. As in the previous section, we use the free energy function $\psi(u, \sigma)$ introduced in the proof of (28).

Lemma 4. If $E>\Gamma$ and the data satisfy (35) 1 , then for all $T>0$

$$
\text { - }\|u(\cdot, T)\|_{L^{2}(\mathbb{R})}+\|v(\cdot, T)\|_{L^{2}(\mathbb{R})}+\|\sigma(\cdot, T)\|_{L^{2}(\mathbb{R})} \leq O(1),
$$$$
\text { - } h\left\|v_{x}\right\|_{L^{2}([0, T] \times \mathbb{R})}^{2}+h\left\|\sigma_{x}\right\|_{L^{2}([0, T] \times \mathbb{R})}^{2} \leq O(1) \text {, }
$$$$
\text { - }\|\sigma-g(u)\|_{L^{2}([0, T] \times \mathbb{R})}^{2} \leq O(\varepsilon) \text {. }
$$

Proof. First $(32)_{3}$ is rewritten as

$$
(\sigma-E u)_{t}=-\frac{1}{\varepsilon}(\sigma-g(u))=-\frac{1}{\varepsilon}(\underbrace{\sigma-E u}_{\alpha}-\underbrace{(g(u)-E u)}_{h(u)}) .
$$

We multiply the equations in (32) by $\sigma, v,\left(u-h^{-1}(\alpha)\right)$, respectively, and use (29) to obtain

$$
\begin{gathered}
\left(\frac{v^{2}}{2}+\psi(u, \sigma)\right)_{t}-(\sigma v)_{x}+\frac{h}{a}\left(\sigma_{x}\right)^{2}+h a\left(v_{x}\right)^{2}+\frac{1}{E \varepsilon}(\sigma-g(u))^{2} \\
\leq\left(\frac{h}{a} \sigma \sigma_{x}+h a v v_{x}\right)_{x} .
\end{gathered}
$$

Then (36] follows from integration over $[0, T] \times \mathbb{R}$. 
Lemma 5. The following identities hold:

$$
\begin{aligned}
& \text { (37) } 1\left\{\begin{array}{r}
\partial_{t}\left(\frac{v^{2}}{2}+G(u)+\varepsilon v v_{t}+\varepsilon h a\left(v_{x}\right)^{2}\right)-\partial_{x}(v g(u)) \\
+\varepsilon\left[a^{2}\left(v_{x}\right)^{2}-\left(v_{t}\right)^{2}\right]+\varepsilon h^{2} a^{2}\left(v_{x x}\right)^{2} \\
+\frac{h}{a} g(u)_{x} \sigma_{x}+h a\left(v_{x}\right)^{2}=\partial_{x}\left(F_{1}\right), \\
F_{1}:=\frac{h}{a} g(u) \sigma_{x}+(\varepsilon a+h) a v v_{x}+2 \varepsilon h a v v_{x t} \\
-\varepsilon h^{2} a^{2} v v_{x x x}+\varepsilon h^{2} a^{2} v_{x} v_{x x},
\end{array}\right. \\
& \text { (37) }_{2}\left\{\begin{array}{c}
\partial_{t}\left(\frac{1}{2}\left(h a+\varepsilon a^{2}\right)\left(v_{x}\right)^{2}+\frac{1}{2} \varepsilon h^{2} a^{2}\left(v_{x x}\right)^{2}+\frac{1}{2} \varepsilon\left(v_{t}\right)^{2}\right) \\
+\left[\left(v_{t}\right)^{2}-g(u)_{x} v_{t}\right]+2 \varepsilon h a\left(v_{x t}\right)^{2}=\partial_{x}\left(F_{2}\right) \\
F_{2}:=(\varepsilon a+h) a v_{t} v_{x}+2 \varepsilon h a v_{t} v_{x t}-\varepsilon h^{2} a^{2}\left(v_{t} v_{x x x}-v_{x t} v_{x x}\right)
\end{array}\right.
\end{aligned}
$$$$
\text { (37) } 3\left\{\begin{array}{c}
\partial_{t}\left(\frac{1}{2} \varepsilon a^{2}\left(u_{x}\right)^{2}-\left(v+\varepsilon \sigma_{x}\right) u_{x}-\frac{1}{2}\left(\varepsilon+\frac{h}{a}\right)\left(v_{x}\right)^{2}\right) \\
+\left(g(u)_{x} u_{x}-\left(v_{x}\right)^{2}\right) \\
-\left(\varepsilon h a+h^{2}\right)\left(v_{x x}\right)^{2}-\frac{\varepsilon h}{a}\left(\sigma_{x x}\right)^{2}+h a u_{x} v_{x x}=\partial_{x}\left(F_{3}\right), \\
F_{3}:=-\left(\varepsilon h a+h^{2}\right) v_{x} v_{x x}-u_{t}\left(v+\varepsilon \sigma_{x}\right)
\end{array}\right.
$$$$
\text { [37) } 4\left\{\begin{array}{c}
\partial_{t}\left(\frac{1}{2 E}\left(\sigma_{x}\right)^{2}+\frac{1}{2}\left(v_{x}\right)^{2}\right)-\partial_{x}\left(\sigma_{x} v_{x}\right) \\
+\frac{1}{E \varepsilon} \sigma_{x}[\sigma-g(u)]_{x}+\frac{h}{a}\left(\sigma_{x x}\right)^{2}+h a\left(v_{x x}\right)^{2}=\partial_{x}\left(F_{4}\right), \\
F_{4}:=\frac{h}{a} \sigma_{x} \sigma_{x x}+h a v_{x} v_{x x}
\end{array}\right.
$$

$$
\text { [37) }_{5}\left\{\begin{array}{c}
\partial_{t}\left(\frac{1}{2}\left(v+\varepsilon \sigma_{x}-\varepsilon E u_{x}\right)^{2}+G(u)-\frac{1}{2} \varepsilon h a\left(v_{x}\right)^{2}\right) \\
-\partial_{x}\left(v g(u)+\varepsilon^{2} E \sigma_{x} v_{x}\right)+h a\left(v_{x}\right)^{2}+\frac{h}{a} g(u)_{x} \sigma_{x}+\varepsilon h a^{3} u_{x} v_{x x} \\
+\varepsilon\left[\left(\sigma_{x}\right)^{2}-g(u)_{x} \sigma_{x}+E g(u)_{x} u_{x}-\left(v_{t}\right)^{2}\right]=\partial_{x}\left(F_{5}\right) \\
F_{5}:=\frac{h}{a} g(u) \sigma_{x}+h a v v_{x}-\operatorname{shav}_{x} v_{t}-\varepsilon^{2} a^{2} v_{x} \sigma_{x}
\end{array}\right.
$$

where

$$
G(u)=\int_{0}^{u} g(s) d s .
$$

Proof. The first identity is obtained if we multiply $(33)_{1}$ by $g(u),(33)_{2}$ by $v$, add the results and rearrange the terms via some integrations by parts.

For the second identity, one has to multiply $(33)_{2}$ by $v_{t}$ and perform a few integrations by parts.

To derive the third identity, we multiply $(33)_{2}$ by $u_{x}$ and use $(32)_{3}$ to arrive at

$$
\begin{aligned}
g^{\prime}(u)\left(u_{x}\right)^{2} & =u_{x}\left(v_{t}-h a v_{x x}-\varepsilon E u_{x t}+\varepsilon \sigma_{x t}\right) \\
& =u_{x}\left(v+\varepsilon \sigma_{x}\right)_{t}-h a u_{x} v_{x x}-\varepsilon a^{2} u_{x} u_{x t} .
\end{aligned}
$$

At this point, we use the equality $e_{x} f_{t}=e_{t} f_{x}-\left(f e_{t}\right)_{x}+\left(f e_{x}\right)_{t}$ for $f=v+\varepsilon \sigma_{x}$, $e=u$. This leads to the identity

$$
\begin{gathered}
\left(\frac{1}{2} \varepsilon E\left(u_{x}\right)^{2}-u_{x}\left(v+\varepsilon \sigma_{x}\right)\right)_{t}+g^{\prime}(u)\left(u_{x}\right)^{2}+h a u_{x} v_{x x} \\
-\left(v_{x}+\frac{h}{a} \sigma_{x x}\right)\left(v_{x}+\varepsilon \sigma_{x x}\right)=-\left[u_{t}\left(v+\varepsilon \sigma_{x}\right)\right]_{x}
\end{gathered}
$$


Then the third equality follows from (38) and the observation that, due to (32) 2 , there holds

$$
v_{x} \sigma_{x x}=\frac{1}{2}\left[\left(v_{x}\right)^{2}\right]_{t}-h a\left(v_{x} v_{x x}\right)_{x}+h a\left(v_{x x}\right)^{2} .
$$

From (34) we have

$$
\left\{\begin{array}{l}
\frac{1}{E} \sigma_{x t}-v_{x x}=\frac{h}{a} \sigma_{x x x}-\frac{1}{\varepsilon E}(\sigma-g(u))_{x} \\
v_{x t}-\sigma_{x x}=h a v_{x x x}
\end{array}\right.
$$

The fourth identity is obtained from multiplications of $(39)_{1}$ by $\sigma_{x}$, of $(39)_{2}$ by $v_{x}$, adding the resulting equations and integrating by parts.

To derive the fifth identity, one adds (37) $)_{1}+\varepsilon E(37)_{3}+\varepsilon^{2} E(37)_{4}$. After rearranging the terms and using (33) 2 , one obtains the desired result.

We introduce the notations

$$
\begin{aligned}
& L_{1}=\frac{1}{2}\left(v+\varepsilon \sigma_{x}-\varepsilon E u_{x}\right)^{2}+G(u)+\frac{\varepsilon^{2}}{2}\left[E\left(v_{x}\right)^{2}+h^{2} E\left(v_{x x}\right)^{2}+\left(v_{t}\right)^{2}\right], \\
& L_{2}=v g(u)+\varepsilon^{2} E \sigma_{x} v_{x} .
\end{aligned}
$$

Combining $\varepsilon(37)_{2}$ and $(37)_{5}$, we get

$$
\begin{aligned}
\left(L_{1}\right)_{t}+\varepsilon & {\left[\left(\sigma_{x}\right)^{2}-2 g(u)_{x} \sigma_{x}+E g^{\prime}(u)\left(u_{x}\right)^{2}\right] } \\
& +2 \varepsilon^{2} h a\left(v_{x t}\right)^{2}+h a\left(v_{x}\right)^{2}+\frac{h}{a} g(u)_{x} \sigma_{x}+\varepsilon h a^{3} u_{x} v_{x x}-\varepsilon h a g(u)_{x} v_{x x} \\
=( & \left(L_{2}+\varepsilon F_{2}+F_{5}\right)_{x} .
\end{aligned}
$$

Observe now that, under the condition $E>g^{\prime}(u)>0$, the quadratic form

$$
\begin{aligned}
Q & =\left(\sigma_{x}\right)^{2}-2 g^{\prime}(u) u_{x} \sigma_{x}+E g^{\prime}(u)\left(u_{x}\right)^{2} \\
& =\left(\sigma_{x}-g(u)_{x}\right)^{2}+g^{\prime}(u)\left(E-g^{\prime}(u)\right)\left(u_{x}\right)^{2} \geq 0
\end{aligned}
$$

is positive definite.

\subsection{Entropy consistency and convergence.}

Lemma 6. Under the assumptions (6), $E>\Gamma$ and (35) the following estimates hold for $h=o(\varepsilon)$ and $T>0$ :

$$
\begin{aligned}
\varepsilon\left\|u_{x}\right\|_{L^{2}(\mathbb{R} \times[0, T])}^{2}+\varepsilon\left\|v_{x}\right\|_{L^{2}(\mathbb{R} \times[0, T])}^{2}+\varepsilon\left\|\sigma_{x}\right\|_{L^{2}(\mathbb{R} \times[0, T])}^{2} & \leq O(1), \\
\varepsilon^{2} h\left\|v_{x x}\right\|_{L^{2}(\mathbb{R} \times[0, T])}^{2}+\varepsilon^{2} h\left\|\sigma_{x x}\right\|_{L^{2}(\mathbb{R} \times[0, T])}^{2} & \leq O(1) .
\end{aligned}
$$

Proof. From (40), we obtain with unambiguous notations

$$
\begin{aligned}
\left(I_{1}\right)_{t}+\left(J_{1}\right)_{x}+\frac{\varepsilon}{O(1)}\left[\left(u_{x}\right)^{2}+\left(\sigma_{x}\right)^{2}\right] \leq & O(1) h\left[\left(u_{x}\right)^{2}+\left(\sigma_{x}\right)^{2}\right] \\
& +O(1) \varepsilon h\left|u_{x} v_{x x}\right|
\end{aligned}
$$

where $I_{1} \geq 0$. From $(\underline{37})_{2}$, we get

$$
\left(I_{2}\right)_{t}+\left(J_{2}\right)_{x}+\frac{\varepsilon}{O(1)}\left(v_{t}\right)^{2} \leq O(1) \varepsilon\left(u_{x}\right)^{2},
$$

where $I_{2} \geq 0$. From $(37)_{4}$, we have

$$
\left(I_{3}\right)_{t}+\left(J_{3}\right)_{x}+\frac{\varepsilon}{O(1)}\left(\sigma_{x}\right)^{2}+\frac{\varepsilon^{2} h}{O(1)}\left[\left(\sigma_{x x}\right)^{2}+\left(v_{x x}\right)^{2}\right] \leq O(1) \varepsilon\left(u_{x}\right)^{2},
$$


where $I_{3} \geq 0$. We add these relations with a large weight factor onto (42) and we obtain

$$
\begin{aligned}
I_{t}+ & J_{x}+\frac{\varepsilon}{O(1)}\left[\left(u_{x}\right)^{2}+\left(\sigma_{x}\right)^{2}+\left(v_{t}\right)^{2}\right]+\frac{\varepsilon^{2} h}{O(1)}\left[\left(\sigma_{x x}\right)^{2}+\left(v_{x x}\right)^{2}\right] \\
\leq & O(1) h\left[\left(u_{x}\right)^{2}+\left(\sigma_{x}\right)^{2}\right]+O(1) \varepsilon h\left|u_{x} v_{x x}\right| .
\end{aligned}
$$

Provided $h=o(\varepsilon)$, the right-hand-side can be absorbed in the left for $\varepsilon$ small enough. Since $I \geq 0$, this yields that

$$
\begin{aligned}
\int_{\mathbb{R} \times[0, T]} \varepsilon & {\left[\left(u_{x}\right)^{2}+\left(\sigma_{x}\right)^{2}+\left(v_{t}\right)^{2}\right]+\varepsilon^{2} h\left[\left(\sigma_{x x}\right)^{2}+\left(v_{x x}\right)^{2}\right] d x d t } \\
\leq O(1) & {\left[\int_{\mathbb{R}}\left(u_{0}\right)^{2}+\left(v_{0}\right)^{2} d x\right.} \\
& \left.+\int_{\mathbb{R}} \varepsilon^{2}\left[\left(u_{0 x}\right)^{2}+\left(v_{0 x}\right)^{2}+\left(\sigma_{0 x}\right)^{2}\right]+\varepsilon^{2} h^{2}\left(v_{0 x x}\right)^{2} d x\right]
\end{aligned}
$$

is bounded in terms of (35). The $L^{1}$ estimate on $\varepsilon v_{x}^{2}$ follows from (37) 1 , (35) and (43).

Lemma 7. Under the assumptions of Lemma 6,

$$
\eta(u, v)_{t}+q(u, v)_{x} \text { lies in a compact of } H_{\text {loc }}^{-1}\left(\mathbb{R} \times \mathbb{R}^{+}\right)
$$

for any entropy-entropy flux pair satisfying (24).

Proof. We consider the system (32) written in the form (33). We use the chain rule for the smooth approximate solutions:

$$
\eta(u, v)_{t}+q(u, v)_{x}=\frac{h}{a} \eta_{u}(u, v) \sigma_{x x}+\eta_{v}(u, v)\left[h a v_{x x}+(\sigma-g(u))_{x}\right] .
$$

We split the right-hand-side the following way:

$$
\begin{gathered}
\partial_{x}\left(\frac{h}{a} \eta_{u}(u, v) \sigma_{x}+\eta_{v}(u, v)\left[h a v_{x}+(\sigma-g(u))\right]\right) \\
-\frac{h}{a} \eta_{u u}(u, v) u_{x} \sigma_{x}-\eta_{u v}(u, v)\left[\frac{h}{a} v_{x} \sigma_{x}+h a u_{x} v_{x}\right]-\eta_{v v}(u, v) h a\left(v_{x}\right)^{2} \\
-[\sigma-g(u)]\left[\eta_{u v}(u, v) u_{x}+\eta_{v v}(u, v) v_{x}\right] \\
\stackrel{\text { def }}{=}\left(\mathcal{I}_{0}\right)_{x}+\mathcal{I}_{1}+\mathcal{I}_{2} .
\end{gathered}
$$

We notice that $\mathcal{I}_{2}$ rewrites

$$
\mathcal{I}_{2}=-\sqrt{\varepsilon}\left[\eta_{u v}(u, v) u_{x}+\eta_{v v}(u, v) v_{x}\right] \frac{\sigma-g(u)}{\sqrt{\varepsilon}} .
$$

Under the assumptions of Lemma $6, \mathcal{I}_{0} \rightarrow 0$ in $L^{2}(\mathbb{R} \times[0, T])$ and $\mathcal{I}_{1}, \mathcal{I}_{2}$ are bounded in $L^{1}(\mathbb{R} \times[0, T])$ for all entropy pairs satisfying (24). Then (44) follows from Murat's lemma [14].

As in Theorem 1 we conclude:

Theorem 3. Let the initial data satisfy (35), let g satisfy (66), (17) and $E>\Gamma$, and consider a family of solutions $\left\{\left(u^{\varepsilon, h}, v^{\varepsilon, h}, \sigma^{\varepsilon, h}\right)\right\}_{\varepsilon, h>0}$ of (33). If $h=o(\varepsilon)$, then, along a subsequence,

$$
u^{\varepsilon, h} \rightarrow u, \quad v^{\varepsilon, h} \rightarrow v, \text { a.e. }(x, t) \text { and in } L_{l o c}^{p}(\mathbb{R} \times(0, T)) \text {, for } p<2,
$$

and $(u, v)$ is a weak solution of (11). 
Remark 2. Theorem 2 guarantees convergence for the semidiscrete scheme in the range $\varepsilon=O(h)$ while Theorem [3] states convergence of the associated relaxationdiffusion system on the complementary range $h=o(\varepsilon)$. The origin of this discrepancy at the level of the proof is the following. For the scheme it is easy to control the numerical derivative of $u_{x}$ (see Remark 1). By contrast, for the relaxation-diffusion system, the energy estimate in Lemma 4 does not yield control of $u_{x}$. In our proof we used the stabilizing control of relaxation to obtain control of $u_{x}$, which leads to the restriction $h=o(\varepsilon)$ stating that relaxation dominates. We do not know if the analytical result for the relaxation-diffusion system is optimal. Regarding the scheme, the numerical experiments in Section 5 suggest that the range $\varepsilon=O(h)$ is optimal and that oscillations develop as we approach the boundary of this parameter range. This indicates that the behavior of the relaxation-diffusion system does not accurately describe the behavior of the relaxing scheme.

\section{NumERICAL EXPERIMENTS}

In this section, we present and discuss some numerical experiments that we obtained for fully discrete versions of the scheme (25). We also display comparisons between this approach and the Jin and Xin algorithm 9 . In all computations, we used a splitting technique to treat the stiff relaxation term. This type of algorithm has been studied, for instance, in [1] in the context of relaxation approximations for one-dimensional scalar conservation laws.

5.1. Comparison with the Jin-Xin scheme for $\varepsilon=h^{2}$. We considered a Riemann problem for (2) with the following initial data:

$$
\left\{\begin{array} { l } 
{ u _ { l } = 2 } \\
{ v _ { l } = 2 } \\
{ \sigma _ { l } = 1 }
\end{array} \text { and } \left\{\begin{array}{l}
u_{r}=1 \\
v_{r}=2 \\
\sigma_{r}=1
\end{array}\right.\right.
$$

We chose $g(u)=u+u^{3}$ and fixed $E=100$. The CFL condition implies that the time step $\Delta t$ and the cell width $h$ have to satisfy the relation $\Delta t \leq h / 20$. Figures 11 2 and 3 display the numerical results obtained for both relaxation schemes with step $h=0.01$ at time $T=0.2$, and also for the associated relaxed schemes (obtained by fixing $\sigma=g(u)$ in (25) $)$. We selected $\varepsilon=h / 100$, in order to let the relaxation process fully operate in each cell of the computational grid. It is clear that a firstorder scheme of the current approach is less diffusive than a first-order Jin-Xin scheme (for which we fixed the parameter $\kappa=\lambda=\sqrt{E}$ so as to use the same CFL numbers).

5.2. Comparisons in the range $\varepsilon \simeq h$. The preceding results ensure the convergence of the relaxation scheme (25) for values of $\varepsilon$ which are of the order of $h$. In practice, one notices easily that oscillations degrade these numerical approximations as soon as a certain value of $\varepsilon / h$ is reached. For example, we illustrate in Figures 4 and 5 the appearance of oscillations in the relaxation term for the problem (45) at $T=0.1$. On the other hand, the relaxation terms involved in the diffusive Jin-Xin approach remain acceptable. This provides a numerical justification of the restriction on the ratio $\varepsilon / h$ made in Theorem 2 ,

On the other hand, the approximations generated by the Jin-Xin approach are more diffusive, but remain stable in any range of parameters $\varepsilon, h$. We illustrate this statement in Figure [6] where the variable $u$ is shown for $h=\varepsilon^{1.01}$ and $0.0002 \leq h \leq$ 0.02 . 


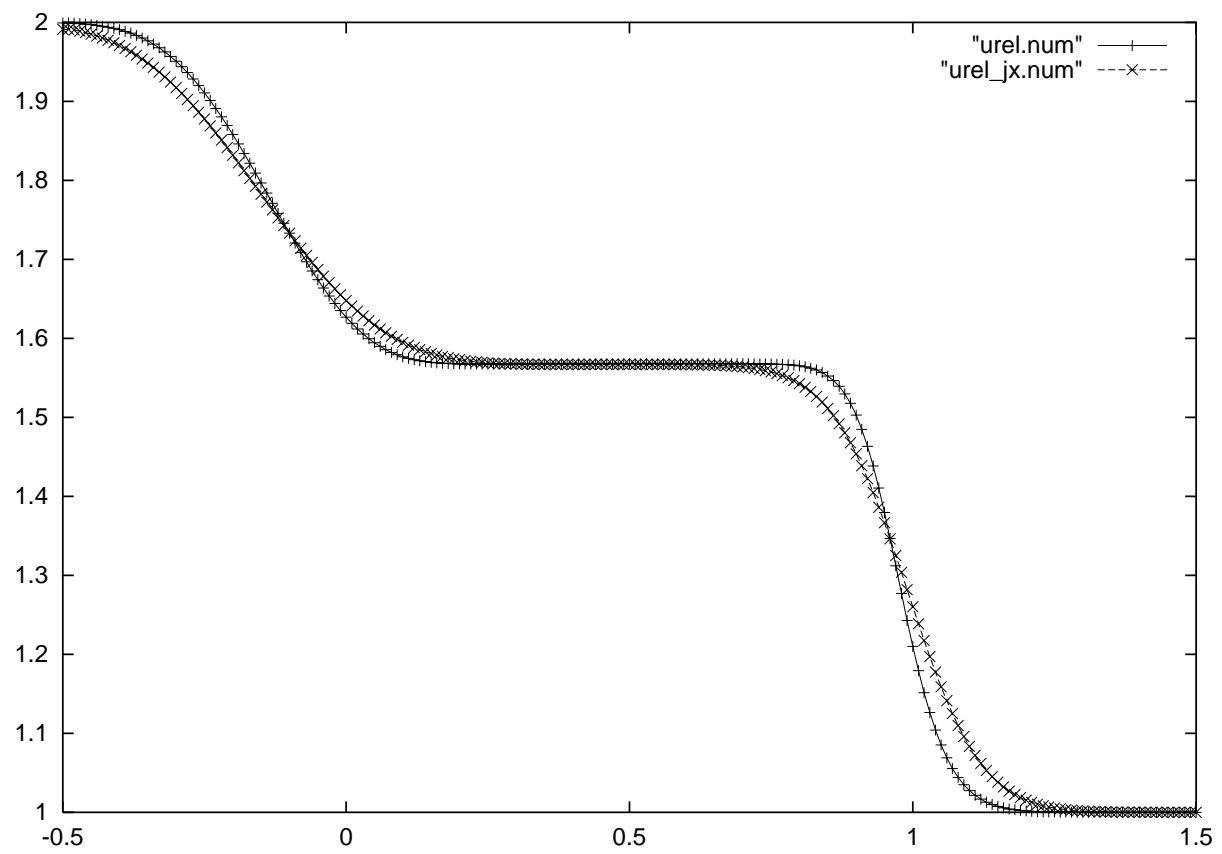

Figure 1. Comparison between Jin-Xin and the proposed relaxation scheme on $u^{h, \varepsilon}$.

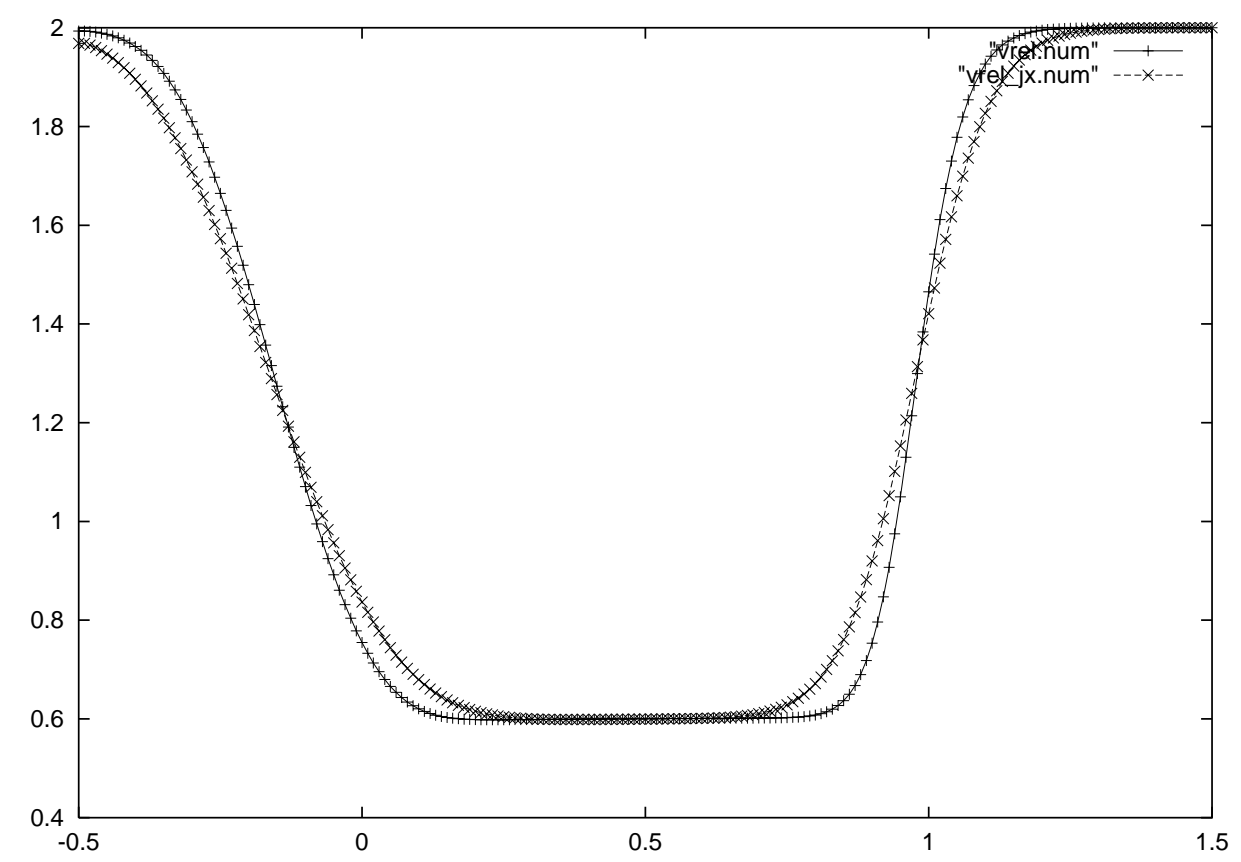

Figure 2. Comparison between Jin-Xin and the proposed relaxation scheme on $v^{h, \varepsilon}$. 


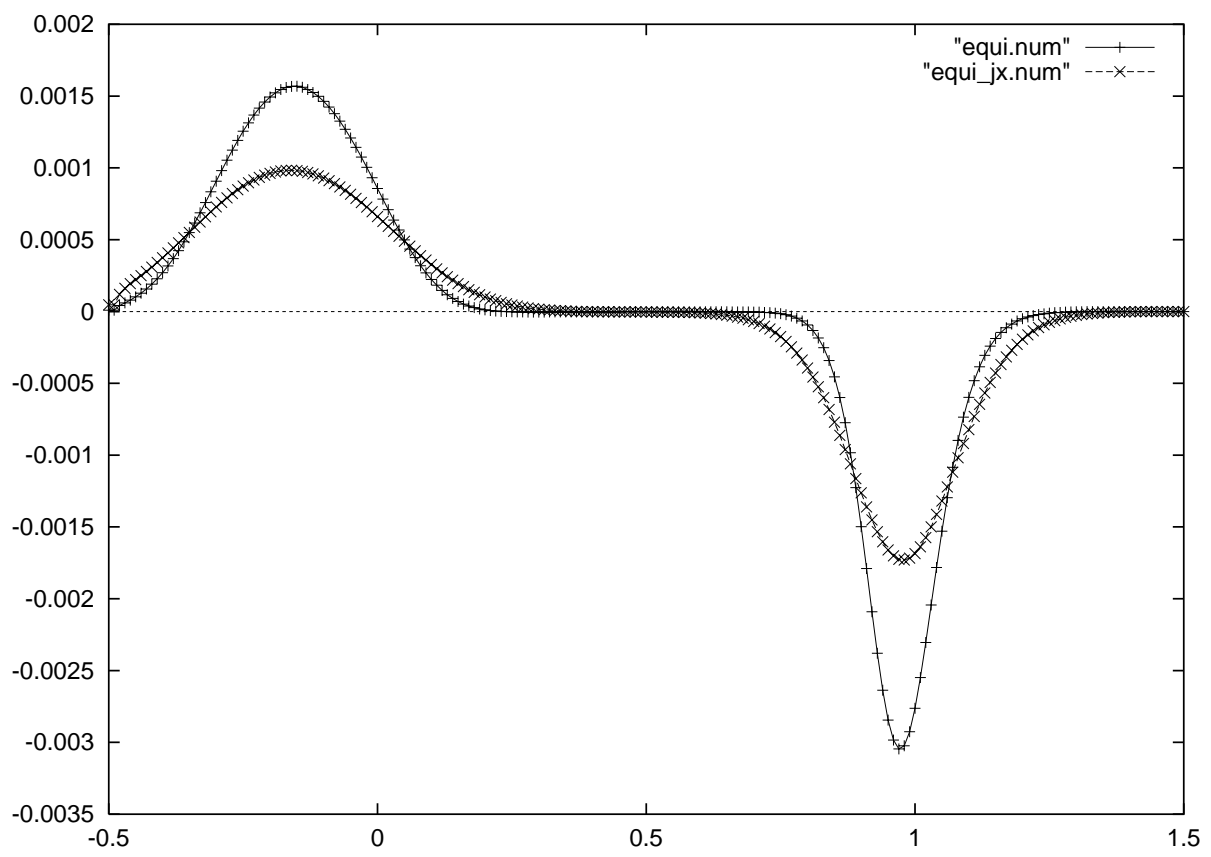

Figure 3. Comparison between Jin-Xin and the proposed relaxation scheme on $\sigma^{h, \varepsilon}-g\left(u^{h, \varepsilon}\right)$.

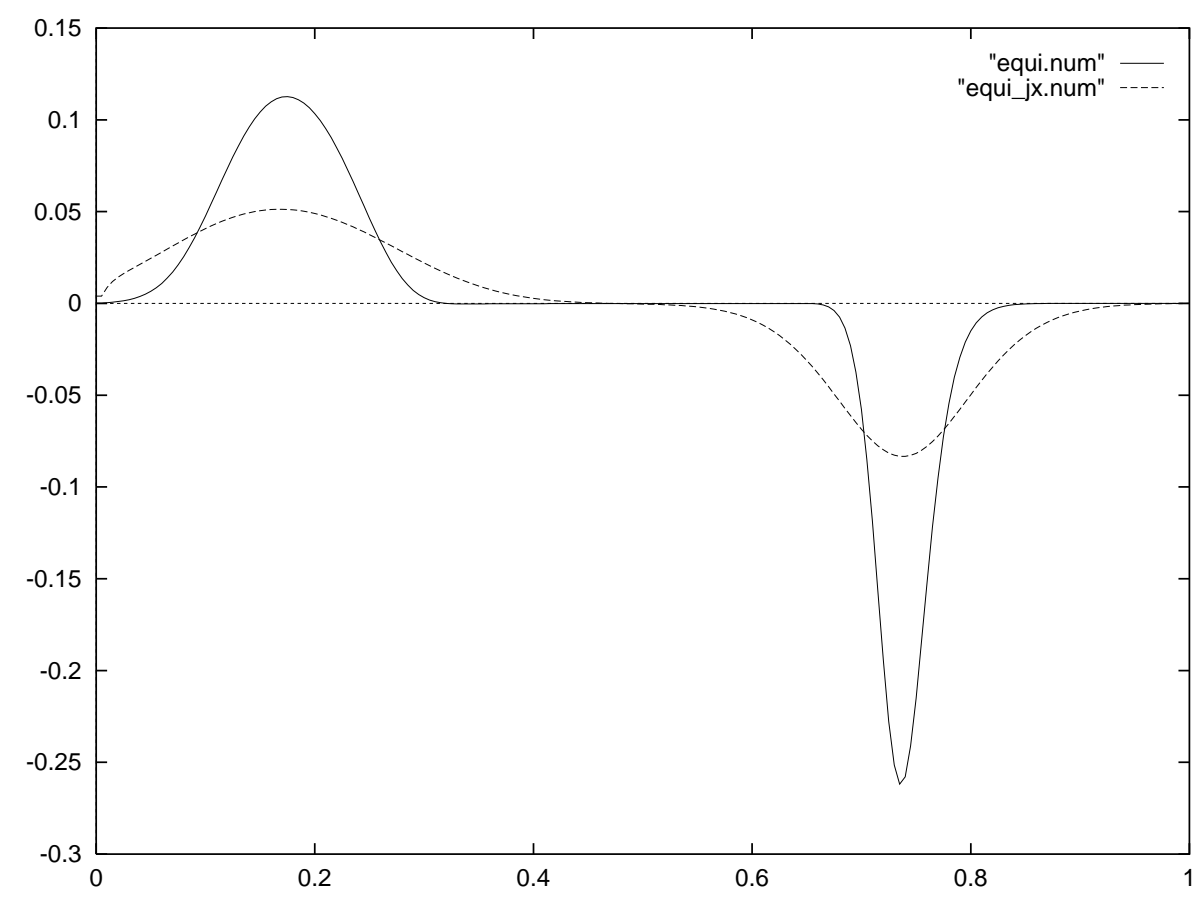

Figure 4. Numerical profiles of the relaxation terms for both schemes with $h=0.005, \varepsilon=0.0002$. 


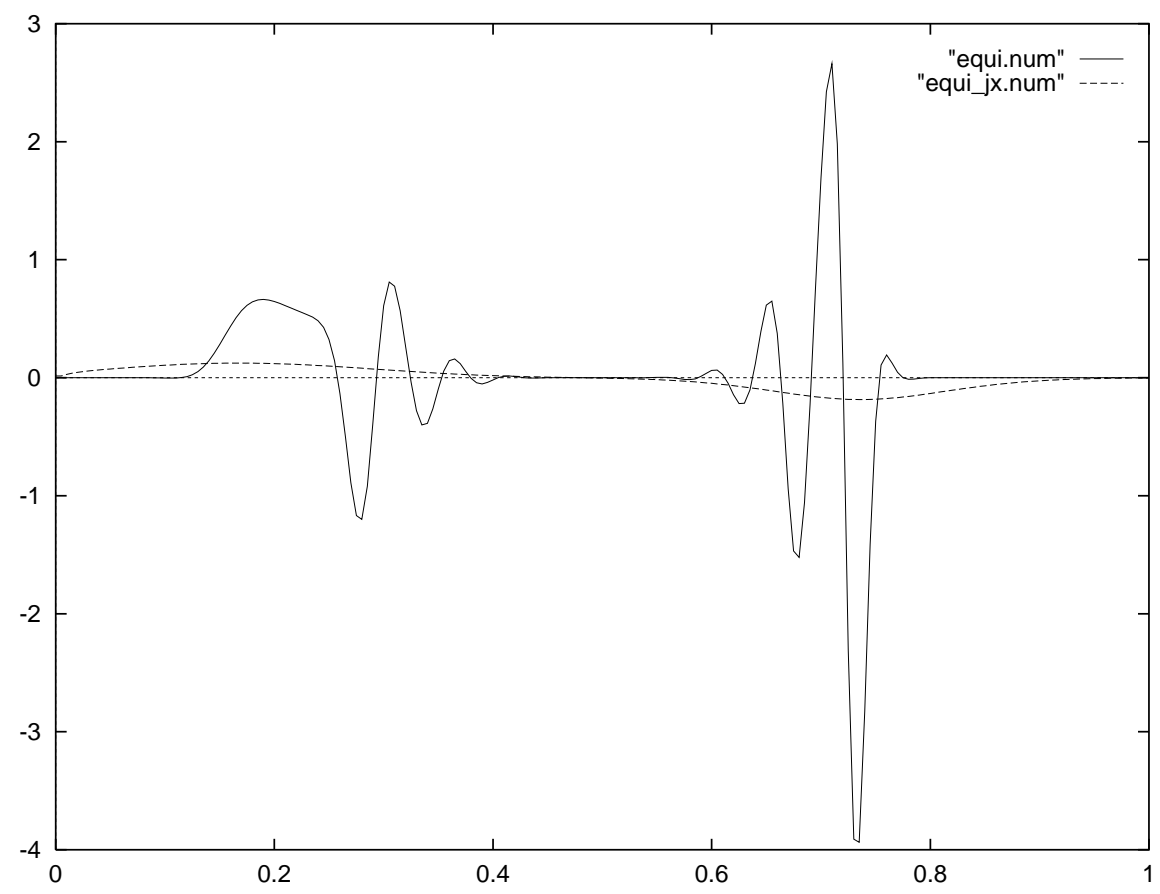

Figure 5. Numerical profiles of the relaxation terms for both schemes with $h=0.005, \varepsilon=0.0004$.

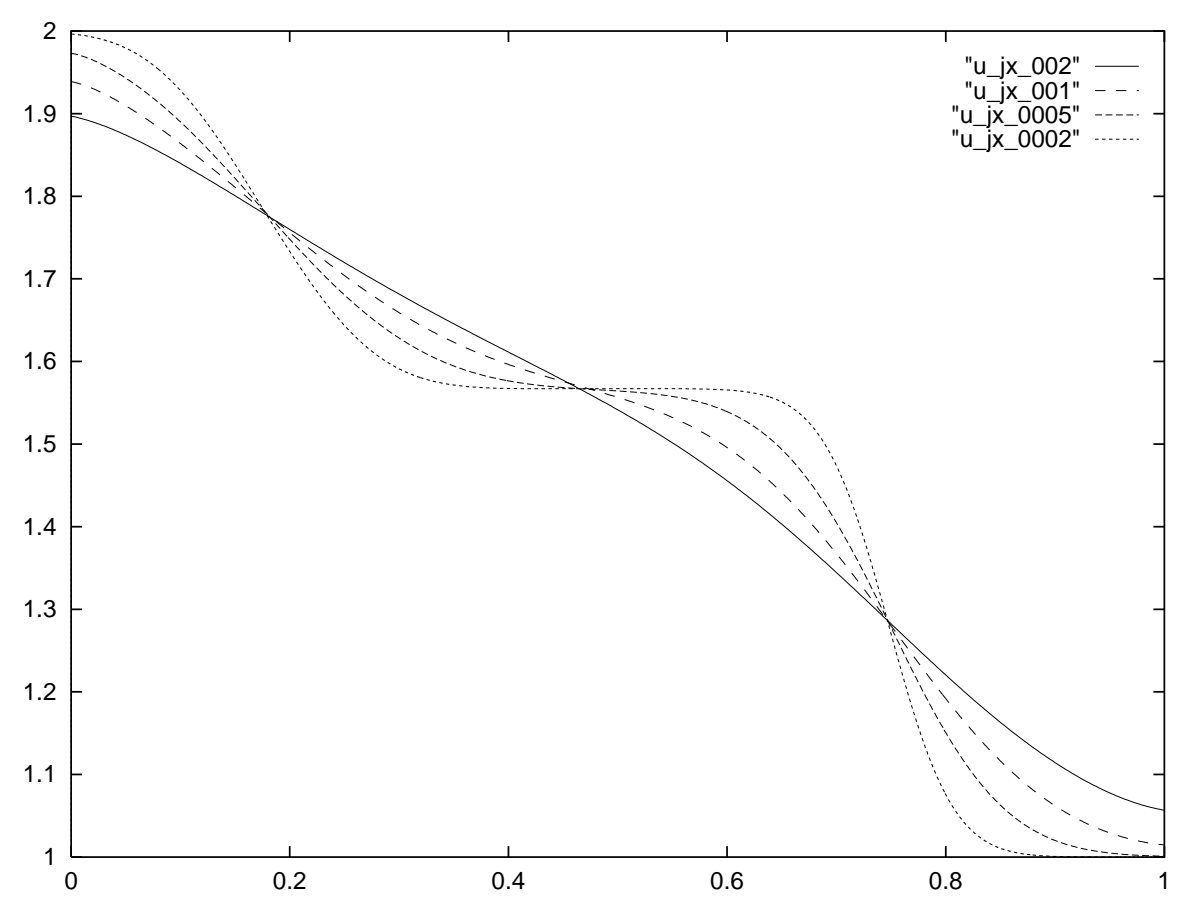

Figure 6. Convergence of the Jin-Xin approximations in the $u$ variable for $h=\varepsilon^{1.01}$. 


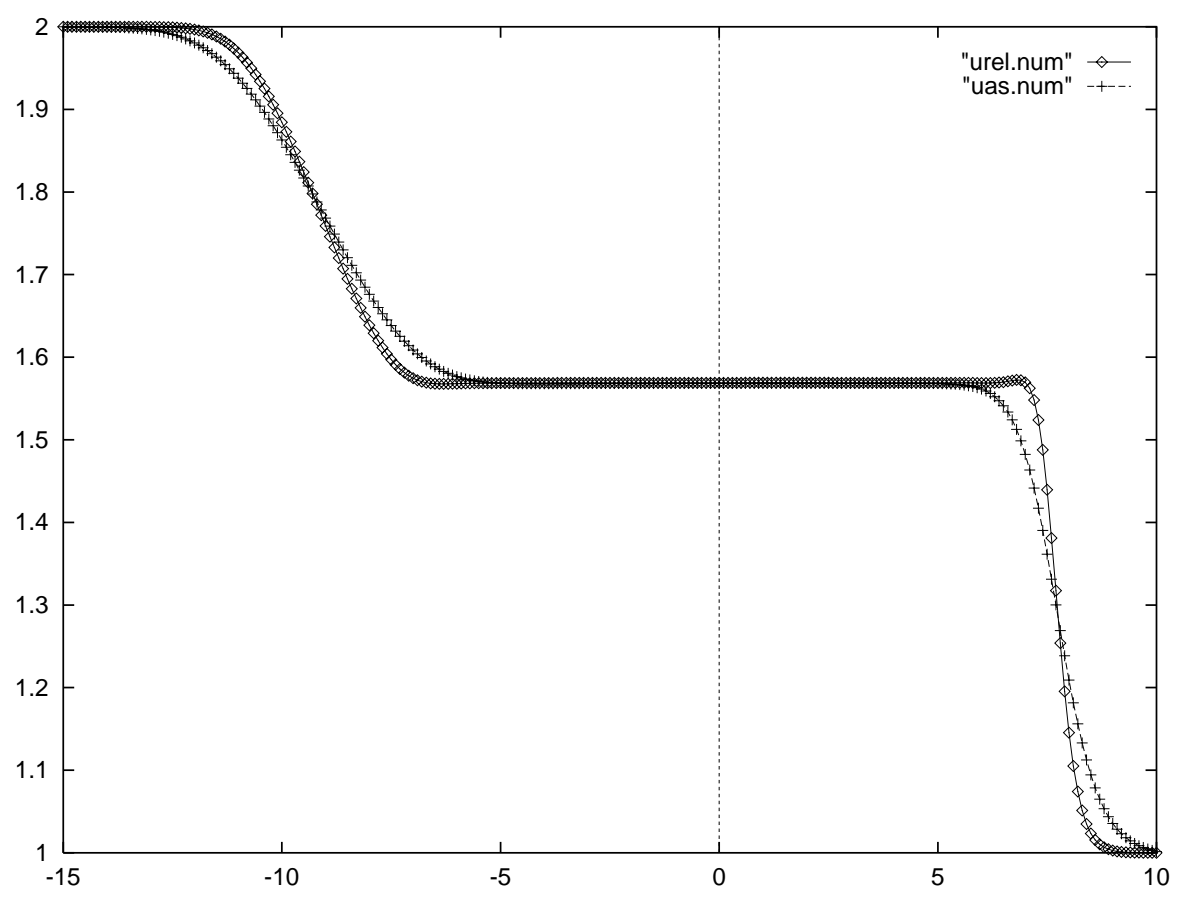

FiguRE 7. Long-time behavior of the proposed approach on $u^{h, \varepsilon}$.

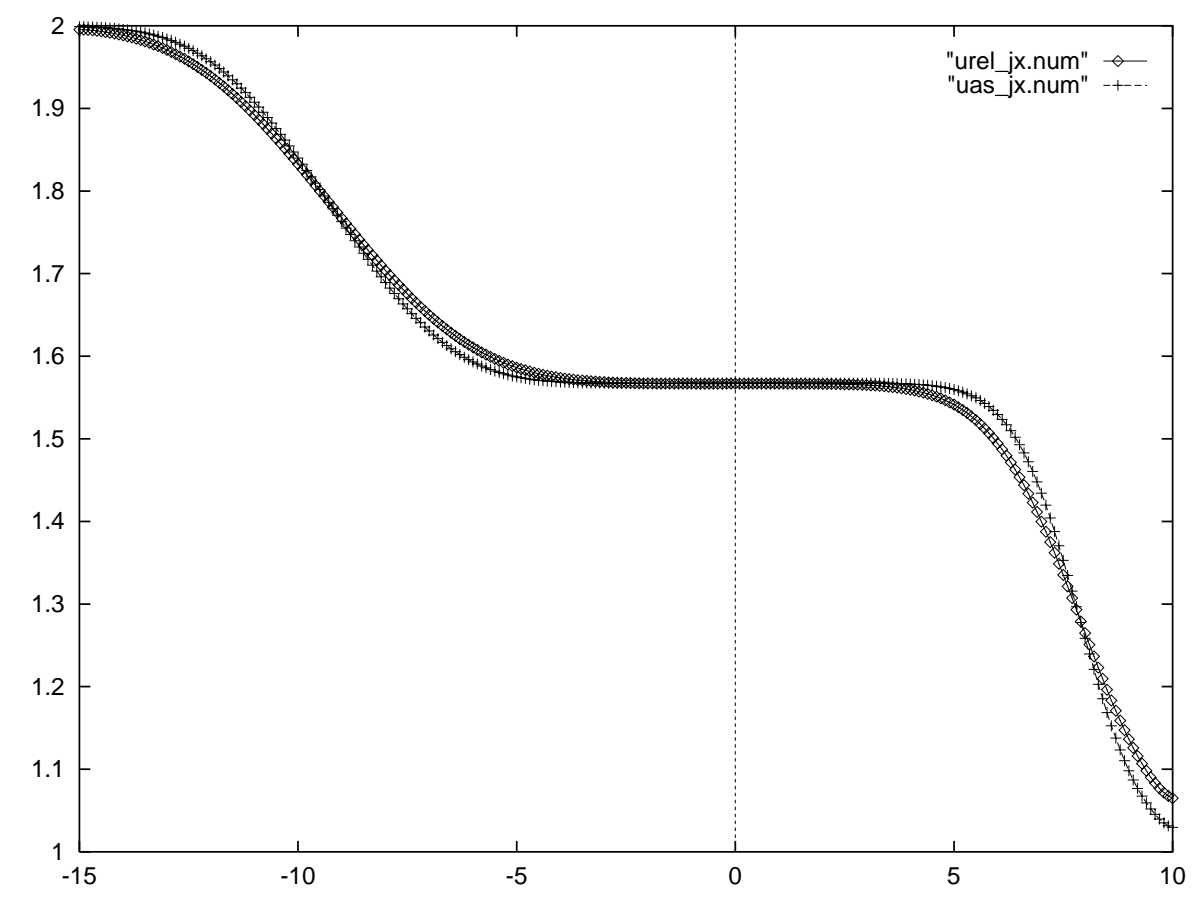

Figure 8. Long-time behavior of the Jin-Xin relaxation scheme on $u^{h, \varepsilon}$. 


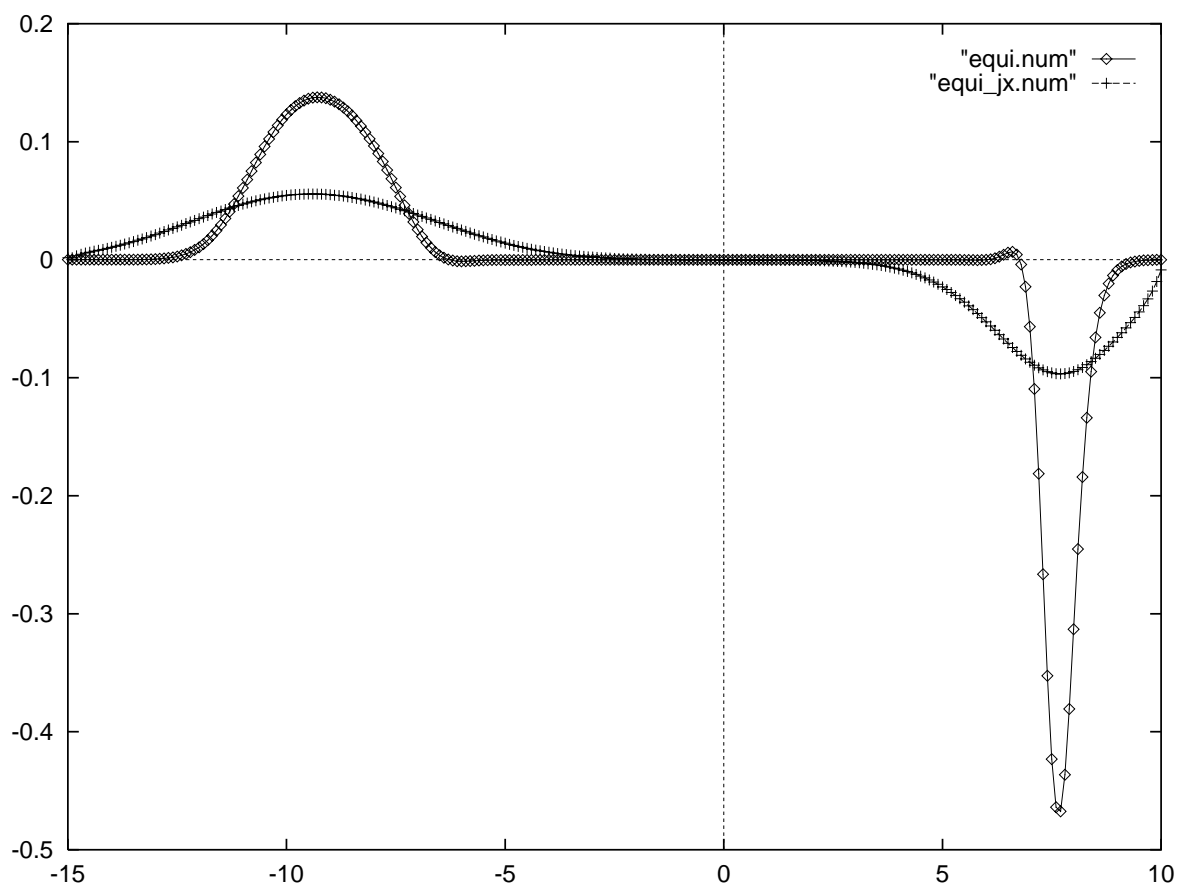

Figure 9. Comparison between Jin-Xin and the proposed relaxation scheme on $\sigma^{h, \varepsilon}-g\left(u^{h, \varepsilon}\right)$.

5.3. Large-time behaviour of the proposed scheme. To check the stability of the numerical wave profiles, we performed a numerical run on a bigger domain and during a longer time $T=3$. In Figures 7 and 8 we display both relaxing and relaxed numerical solutions for the two possible relaxation schemes. In Figure 9 we compare the values of the relaxation term for both schemes. We fixed $h=0.1$, $\varepsilon=0.005$ and used the same Riemann problem (45) and the same CFL restrictions.

\section{REFERENCES}

[1] D. Aregba-Driollet and R. Natalini, Convergence of relaxation schemes for conservation laws, Appl. Anal. 61 (1996), 163-193. CMP 98:13

[2] D. Aregba-Driollet and R. Natalini, Discrete kinetic schemes for multi-dimensional conservation laws, (1998), (preprint).

[3] H. Brezis, Analyse Fonctionnelle, Théorie et Applications Masson (1983). MR 85a:46001

[4] G.-Q. Chen, C.D Levermore and T.-P. Liu, Hyperbolic conservation laws with stiff relaxation terms and entropy, Comm. Pure Appl. Math. 47 (1994), 789-830. MR 95h:35133

[5] F. Coquel and B. Perthame, Relaxation of energy and approximate Riemann solvers for general pressure laws in fluid dynamics, SIAM J. Numer. Anal. 35 (1998), 2223-2249. MR 2000a:76129

[6] R. DiPerna, Convergence of approximate solutions to conservation laws, Arch. Rational Mech. Analysis 82 (1983), 27-70. MR 84k:35091

[7] C. Faciu and M. Mihailescu-Suliciu, The energy in one-dimensional rate-type semilinear viscoelasticity, Int. J. Solids Structures 23 (1987), 1505-1520. MR 89a:73032

[8] D. Henry, Geometric theory of semilinear parabolic equations, Lecture Notes in Math. 840, Springer, New York, 1981. MR 83j:35084

[9] S. Jin and Z. Xin, The relaxation schemes for systems of conservation laws in arbitrary space dimensions, Comm. Pure Appl. Math. 48 (1995), 235-277. MR 96c:65134 
[10] M. Katsoulakis and A. Tzavaras, Contractive relaxation systems and the scalar multidimensional conservation law, Comm. Partial Differential Equations 22 (1997), 195-233. MR 97m:35168

[11] M. Katsoulakis, G. Kossioris G. and Ch. Makridakis, Convergence and error estimates of relaxation schemes for multidimensional conservation laws, Comm. Partial Differential Equations 24 (1999), 395-424. CMP 99:11

[12] C. Lattanzio and D. Serre, Convergence of a relaxation scheme for $N \times N$ hyperbolic systems of conservation laws, (preprint).

[13] Y.G. Lu and C. Klingenberg, The Cauchy problem for hyperbolic conservation laws with three equations, J. Math. Anal. Appl. 202 (1996) 206-216. MR 97h:35147

[14] F. Murat, L'injection du cône positif de $H^{-1}$ dans $W^{-1, q}$ est compacte pour tout $q<2, J$. Math. Pures Appl. 60 (1981), 309-322. MR 83b:46045

[15] R. Natalini, Convergence to equilibrium for the relaxation approximations of conservation laws, Comm. Pure Appl. Math. 49 (1996), 795-823. MR 97c:35131

[16] B. Perthame, An introduction to kinetic schemes for gas dynamics. In An Introduction to Recent Developments in Theory and Numerics for Conservation Laws, D. Kroener, M. Ohlberger and C. Rohde, eds, Lecture Notes in Comp. Science and Engin., Vol. 5, Springer, Berlin, 1998, pp. 1-27.

[17] D. Serre, Relaxation semi-linéaire et cinétique des systèmes de lois de conservation, Ann. Inst. H. Poincaré, Anal. Non Linèaire (to appear).

[18] D. Serre and J. Shearer, Convergence with physical viscosity for nonlinear elasticity, (1993) (preprint).

[19] J. Shearer, Global existence and compactness in $L^{p}$ for the quasi-linear wave equation, Comm. Partial Differential Equations 19 (1994), 1829-1877. MR 95m:35120

[20] L. Tartar, Compensated compactness and applications to partial differential equations. In Nonlinear Analysis and Mechanics - Heriot-Watt symposium - vol IV, R.J. Knops, ed, Pitman Research Notes in Mathematics, New York (1979), pp. 136-192. MR 81m:35014

[21] A. Tveito and R. Winther, On the rate of convergence to equilibrium for a system of conservation laws including a relaxation term, SIAM J. Math. Anal. 28 (1997), 136-161. MR 98a:35087

[22] A. Tzavaras, Viscosity and relaxation approximations for hyperbolic systems of conservation laws. In An Introduction to Recent Developments in Theory and Numerics for Conservation Laws, D. Kroener, M. Ohlberger and C. Rohde, eds, Lecture Notes in Comp. Science and Engin., Vol. 5, Springer, Berlin, 1998, pp. 73-122.

[23] A. Tzavaras, Materials with internal variables and relaxation to conservation laws, Arch. Rational Mech. Anal. 146 (1999), 129-155. CMP 2000:03

Foundation for Research and Technology Hellas / Institute of Applied and Computational Mathematics, P.O. Box 1527, 71110 Heraklion, Crete, Greece

E-mail address: laurent@palamida.math.uch.gr

Department of Mathematics, University of Wisconsin, Madison, Wisconsin 53706

E-mail address: tzavaras@math.wisc.edu 